\title{
Elastic orthorhombic anisotropic parameter inversion: An analysis of parameterization
}

\author{
Ju-Won $\mathrm{Oh}^{1}$ and Tariq Alkhalifah ${ }^{1}$
}

\begin{abstract}
The resolution of a multiparameter full-waveform inversion (FWI) is highly influenced by the parameterization used in the inversion algorithm, as well as the data quality and the sensitivity of the data to the elastic parameters because the scattering patterns of the partial derivative wavefields (PDWs) vary with parameterization. For this reason, it is important to identify an optimal parameterization for elastic orthorhombic FWI by analyzing the radiation patterns of the PDWs for many reasonable model parameterizations. We have promoted a parameterization that allows for the separation of the anisotropic properties in the radiation patterns. The central parameter of this parameterization is the horizontal P-wave velocity, with an isotropic scattering potential, influencing the data at all scales and directions. This parameterization decouples the influence of the scattering potential given by the P-wave velocity perturbation from the polar changes described
\end{abstract}

by two dimensionless parameter perturbations and from the azimuthal variation given by three additional dimensionless parameters perturbations. In addition, the scattering potentials of the $\mathrm{P}$-wave velocity perturbation are also decoupled from the elastic influences given by one S-wave velocity and two additional dimensionless parameter perturbations. The vertical S-wave velocity is chosen with the best resolution obtained from S-wave reflections and converted waves, and little influence on P-waves in conventional surface seismic acquisition. The influence of the density on observed data can be absorbed by one anisotropic parameter that has a similar radiation pattern. The additional seven dimensionless parameters describe the polar and azimuth variations in the P- and S-waves that we may acquire, with some of the parameters having distinct influences on the recorded data on the earth's surface. These characteristics of the new parameterization offer the potential for a multistage inversion from high symmetry anisotropy to lower symmetry ones.

\section{INTRODUCTION}

Accurate interpretations of seismic data acquired in complicated structures might require considering the anisotropic properties of subsurface media. Accordingly, several studies focused on our ability to recover the anisotropic parameters and seismic velocities using full-waveform inversion (FWI) (Virieux and Operto, 2009; Operto et al., 2013; Alkhalifah, 2015a). However, the multiparameter FWI for anisotropic media is prone to entrapment in local minima because we invert for a large number of parameters from given seismic data, which results in possible tradeoffs between parameters (Gholami et al., 2013a). Accordingly, it is difficult to recover the physical properties of anisotropic media using conventional FWI techniques in which we invert for all the parameters at once. To overcome this limitation in anisotropic FWI, particularly for vertical transverse isotropic (VTI) media, Alkhalifah and Plessix (2014) among others introduce alternative parameterizations of the VTI media, and they justify through a scattering angle sensitivity analysis.

Many parameterization studies have focused on the acoustic assumption (Plessix and Cao, 2011; Gholami et al., 2013a, 2013b; Alkhalifah and Plessix, 2014; Alkhalifah 2016), whereas studies covering elastic anisotropy were driven by the inversion application (Koo et al., 2010; Lee et al., 2010; Kamath and Tsvankin, 2013; Oh and Min, 2014). Prieux et al. (2013a) investigate the scattering patterns of parameters resulting from various parameterizations of the acoustic multiparameter FWI and propose a hierarchical inversion strategy for viscoacoustic FWI, which inverts the P-wave velocity, density, and attenuation in two stages. Similar types of analyses have been used to identify an optimal parameterization for acoustic

\footnotetext{
Manuscript received by the Editor 26 November 2015; revised manuscript received 16 June 2016; published online 13 September 2016.

${ }^{1}$ King Abdullah University of Science and Technology, Physical Science and Engineering Division, Thuwal, Kingdom of Saudi Arabia. E-mail: juwon.oh@ kaust.edu.sa; tariq.alkhalifah@kaust.edu.sa.

(C) 2016 Society of Exploration Geophysicists. All rights reserved.
} 
VTI FWI (Gholami et al., 2013a, 2013b; Alkhalifah and Plessix, 2014).

Regarding elastic FWI, Köhn et al. (2012) compare the influences of the parameterization in isotropic elastic media. Prieux et al. (2013b) propose an optimal workflow for viscoelastic FWI and apply their workflow to the multicomponent ocean-bottom-cable (OBC) data from the Valhall field in the North Sea. Oh and Min (2014) suggest a new parameterization using Poisson's ratio for elastic VTI media by analyzing the source mechanism of the virtual source. Through these efforts, the VTI FWI has been improved regardless of the types of wave equations. However, recently, an FWI that focuses on more realistic anisotropic media seems achievable as improvements in the computational power and data quality seem to allow for that. Köhn et al. (2015) show a sensitivity analysis of 21 elastic constants in triclinic anisotropic media taking into consideration various acquisition systems and configurations. For elastic orthorhombic media, they conclude that it will be very hard to recover all of the parameters needed to describe such anisotropy (especially, when the data offset is limited compared with the target depth).

Alkhalifah (2016) highlights the power of the horizontal P-wave velocity in reducing the number of parameters we need to invert for in VTI media, especially when combined with the proper dimensionless parameters. For orthorhombic media, Masmoudi and Alkhalifah (2016) use that insight and propose a new parameterization for acoustic orthorhombic anisotropy by suggesting two deviation parameters to the acoustic VTI parameterization that allows the azimuth variation to be decoupled from the polar variation with its own parameters offering a multistage inversion possibility. Based on the P-P radiation patterns and traveltime sensitivity of six acoustic orthorhombic parameters, they show that the number of parameters we need to invert for in acoustic orthorhombic media can be reduced to three.

In this paper, we extend the study to elastic orthorhombic media and derive radiation patterns for P-waves, as well as SV- and $\mathrm{SH}$ waves, and possible conversions in between. We demonstrate with the new parameterization that we can easily upscale from the acoustic to the elastic case, as we add more components of the observed data into the inversion. We also demonstrate that the new parameterization also provides good separation of anisotropy based on the parameter class, such as isotropic parameters, VTI parameters, and additional HTI parameters. We specifically focus this study on the radiation patterns of the partial derivative wavefields (PDWs) for particular parameterizations, which provide better insights into the source mechanism of the virtual source in the $3 \mathrm{D}$ elastic orthorhombic media.

\section{RADIATION PATTERN OF PDWs}

\section{Definition of the gradient direction}

Compared with a monoparameter FWI, multiparameter FWI is more complicated because we need to consider several physical parameters (so-called parameter class) at the same nodal point in the model grid, which results in highly dimensional models. The number of model parameters increases as we take into account more realistic physics, such as elasticity, viscosity, and anisotropy. In the 3D elastic orthorhombic case, the number of parameters we consider, including the density, is 10 .

The gradient direction that minimizes the least-squares norm of the data residual $u_{s}-d_{s}$ is the zero-lag crosscorrelations between the
PDWs (so-called Jacobian) and the data residual as follows (Operto et al., 2013):

$$
\frac{\partial E}{\partial p_{m, n}}=\sum_{s} \sum_{r} \sum_{c}\left[\int J_{s, m, n}^{c}(r, t, \mathbf{p})\left[d_{s}^{c}(r, t)-u_{s}^{c}(r, t, \mathbf{p})\right] d t\right],
$$

where $u_{s}$ and $d_{s}$ denote the modeled and observed seismic data, respectively, and $s, r, c$, and $t$ denote the source, receiver, recording components, and time, respectively; $\mathbf{p}$ represents the model parameter vector that includes 10 independent elastic orthorhombic parameters at every nodal point; $m$ and $n$ indicate the parameter class and the parameter location on nodal points.

The function $J_{s, m, n}^{c}(r, t, \mathbf{p})$ is the PDW with respect to a desired elastic orthorhombic parameter $m$ at the $n$th nodal point. As we mentioned, the gradient direction is defined as the zero-lag crosscorrelation between the PDW and residual wavefields. This means that the radiation pattern (angular dependency of the amplitude of wavefields) of the PDW determines the direction and degree of update for each parameter at each nodal point. Depending on how we parameterize the elastic-wave equation, the radiation patterns of the PDW are affected. Therefore, the analysis of the radiation patterns of the PDW for various parameterizations is crucial to reduce the eventual tradeoff and isolate the invertible parameters. The radiation patterns for the PDW provide insights into which part of the data we can use to invert for each parameter (Operto et al., 2013).

\section{The total radiation response of PDWs}

Orthorhombic anisotropic media (Tsvankin, 1997) have three mutually orthogonal planes, which can be regarded as a combination of VTI and horizontal transverse isotropic (HTI) media. These additional HTI characteristics originate from vertical cracks and lateral regional tectonic stresses.

Here, we show how to derive analytic radiation patterns of the PDW using the 3D orthorhombic elastic-wave equations given by

$$
\rho \frac{\partial^{2} \mathbf{u}}{\partial t^{2}}=\nabla \cdot \boldsymbol{\sigma}+\mathbf{f}_{\mathbf{s}}
$$

and

$$
\boldsymbol{\sigma}=\mathbf{C} \varepsilon
$$

where $\rho$ and $f_{\mathrm{s}}$ denote the density and the seismic source, respectively, and $\mathbf{u}, \boldsymbol{\sigma}$, and $\varepsilon$ indicate the displacement, stress, and strain vectors, respectively. The vectors written in Voigt notation are defined as

$$
\begin{gathered}
\mathbf{u}=\left(\begin{array}{llll}
u_{x} & u_{y} & u_{z}
\end{array}\right)^{T}, \\
\boldsymbol{\sigma}=\left(\begin{array}{llllll}
\sigma_{x x} & \sigma_{y y} & \sigma_{z z} & \sigma_{y z} & \sigma_{x z} & \sigma_{x y}
\end{array}\right)^{T},
\end{gathered}
$$

and

$$
\boldsymbol{\varepsilon}=\left(\begin{array}{llllll}
\varepsilon_{x x} & \varepsilon_{y y} & \varepsilon_{z z} & 2 \varepsilon_{y z} & 2 \varepsilon_{x z} & 2 \varepsilon_{x y}
\end{array}\right)^{T} .
$$

The matrix $\mathbf{C}$ is the stiffness tensor in a Voigt notation as follows: 


$$
\mathbf{C}=\left(\begin{array}{cccccc}
C_{11} & C_{12} & C_{13} & 0 & 0 & 0 \\
C_{12} & C_{22} & C_{23} & 0 & 0 & 0 \\
C_{13} & C_{23} & C_{33} & 0 & 0 & 0 \\
0 & 0 & 0 & C_{44} & 0 & 0 \\
0 & 0 & 0 & 0 & C_{55} & 0 \\
0 & 0 & 0 & 0 & 0 & C_{66}
\end{array}\right)
$$

By taking the partial derivative of elastic-wave equations with respect to the model parameters $p_{m, n}$, we obtain the elastic-wave equations for the PDW in the same form as equation 2 :

$\rho \frac{\partial^{2}}{\partial t^{2}}\left(\frac{\partial \mathbf{u}}{\partial p_{m, n}}\right)=\nabla \cdot\left(\frac{\partial \boldsymbol{\sigma}}{\partial p_{m, n}}\right)+\nabla \cdot\left(\frac{\partial \mathbf{C}}{\partial p_{m, n}} \boldsymbol{\varepsilon}\right)-\left(\frac{\partial \rho}{\partial p_{m, n}} \frac{\partial^{2} \mathbf{u}}{\partial t^{2}}\right)$.

The last two terms in the right side denote the virtual sources, which are generated by a model parameter perturbation and act as a source for the PDW. The first term acts as a point moment tensor source (Oh and Alkhalifah, 2015, 2016), whereas the second term acts as a point source.

Based on the fact that the PDW is governed by the same elasticwave equation as the original wavefields, as equations 2 and 8 show, the PDW in terms of the displacement fields can be approximated as follows (Aki and Richards [1980], p. 111):

$$
\begin{aligned}
& \mathbf{u}_{\mathrm{PDW}(m, Q)}^{\mathrm{P}-\mathrm{P}}\left(\theta_{i}, \phi_{i}, \theta_{d}, \phi_{d}\right) \approx \mathbb{R}_{s}^{\mathrm{P}}\left[\left(\mathbf{e}_{\mathrm{P}_{d}}^{T} \mathbf{M}_{m}^{P_{i}, Q} \mathbf{e}_{\mathrm{P}_{d}}\right)\right. \\
& \left.\quad+\left(\mathbf{e}_{\mathrm{P}_{d}}^{T} \mathbf{f}_{m}^{\mathrm{P}_{i}, Q}\right)\right] \mathbf{e}_{\mathrm{P}_{d}}=\mathbb{R}_{S}^{\mathrm{P}}\left[\mathbb{R}_{m, Q}^{\mathrm{P}-\mathrm{P}}\right] \mathbf{e}_{\mathrm{P}_{d}},
\end{aligned}
$$

$$
\begin{aligned}
& \mathbf{u}_{\mathrm{PDW}(m, Q)}^{\mathrm{P}-\mathrm{SV}}\left(\theta_{i}, \phi_{i}, \theta_{d}, \phi_{d}\right) \approx \mathbb{R}_{s}^{\mathrm{P}}\left[\left(\mathbf{e}_{\mathrm{SV}_{d}}^{T} \mathbf{M}_{m}^{P_{i}, Q} \mathbf{e}_{\mathrm{P}_{d}}\right)\right. \\
& \left.+\left(\mathbf{e}_{\mathrm{SV}_{d}}^{T} \mathbf{f}_{m}^{\mathrm{P}_{i}, Q}\right)\right] \mathbf{e}_{\mathrm{SV}_{d}}=\mathbb{R}_{S}^{\mathrm{P}}\left[\mathbb{R}_{m, Q}^{\mathrm{P}-\mathrm{SV}}\right] \mathbf{e}_{\mathrm{SV}_{d}},
\end{aligned}
$$

$$
\begin{aligned}
& \mathbf{u}_{\mathrm{PDW}(m, Q)}^{\mathrm{P}-\mathrm{SH}}\left(\theta_{i}, \phi_{i}, \theta_{d}, \phi_{d}\right) \approx \mathbb{R}_{s}^{\mathrm{P}}\left[\left(\mathbf{e}_{\mathrm{SH}_{d}}^{T} \mathbf{M}_{m}^{\mathrm{P}_{i}, Q} \mathbf{e}_{\mathrm{P}_{d}}\right)\right. \\
& \left.+\left(\mathbf{e}_{\mathrm{SH}_{d}}^{T} \mathbf{f}_{m}^{\mathrm{P}_{i}, Q}\right)\right] \mathbf{e}_{\mathrm{SH}_{d}}=\mathbb{R}_{s}^{\mathrm{P}}\left[\mathbb{R}_{m, Q}^{\mathrm{P}-\mathrm{SH}}\right] \mathbf{e}_{\mathrm{SH}_{d}},
\end{aligned}
$$

$$
\begin{aligned}
& \mathbf{u}_{\mathrm{PDW}(m, Q)}^{\mathrm{SV}-\mathrm{SV}}\left(\theta_{i}, \phi_{i}, \theta_{d}, \phi_{d}\right) \approx \mathbb{R}_{s}^{\mathrm{SV}}\left[\left(\mathbf{e}_{\mathrm{SV}_{d}}^{T} \mathbf{M}_{m}^{\mathrm{SV}_{i}, Q} \mathbf{e}_{\mathrm{P}_{d}}\right)\right. \\
& \left.+\left(\mathbf{e}_{\mathrm{SV}_{d}}^{T} \mathbf{f}_{m}^{\mathrm{SV}_{i}, Q}\right)\right] \mathbf{e}_{\mathrm{SV}_{d}}=\mathbb{R}_{s}^{\mathrm{SV}}\left[\mathbb{R}_{m, Q}^{\mathrm{SV}-\mathrm{SV}}\right] \mathbf{e}_{\mathrm{SV}_{d}},
\end{aligned}
$$

$$
\begin{gathered}
\mathbf{u}_{\mathrm{PDW}(m \cdot Q)}^{\mathrm{SV}-\mathrm{SH}}\left(\theta_{i}, \phi_{i}, \theta_{d}, \phi_{d}\right) \approx \mathbb{R}_{s}^{\mathrm{SV}}\left[\left(\mathbf{e}_{\mathrm{SH}_{d}}^{T} \mathbf{M}_{m}^{\mathrm{SV}_{i}, Q} \mathbf{e}_{\mathrm{P}_{d}}\right)\right. \\
\left.+\left(\mathbf{e}_{\mathrm{SH}_{d}}^{T} \mathbf{f}_{m}^{\mathrm{SV}_{i}, Q}\right)\right] \mathbf{e}_{\mathrm{SH}_{d}}=\mathbb{R}_{s}^{\mathrm{SV}}\left[\mathbb{R}_{m, Q}^{\mathrm{SV}-\mathrm{SH}}\right] \mathbf{e}_{\mathrm{SH}_{d}},
\end{gathered}
$$

and

$$
\begin{gathered}
\mathbf{u}_{\mathrm{PDW}(m . Q)}^{\mathrm{SH}-\mathrm{SH}}\left(\theta_{i}, \phi_{i}, \theta_{d}, \phi_{d}\right) \approx \mathbb{R}_{s}^{\mathrm{SH}}\left[\left(\mathbf{e}_{\mathrm{SH}_{d}}^{T} \mathbf{M}_{m}^{\mathrm{SH}_{i}, Q} \mathbf{e}_{\mathrm{P}_{d}}\right)\right. \\
\left.+\left(\mathbf{e}_{\mathrm{SH}_{d}}^{T} \mathbf{f}_{m}^{\mathrm{SH}_{i}, Q}\right)\right] \mathbf{e}_{\mathrm{SH}_{d}}=\mathbb{R}_{s}^{\mathrm{SH}}\left[\mathbb{R}_{m, Q}^{\mathrm{SH}-\mathrm{SH}}\right] \mathbf{e}_{\mathrm{SH}_{d}},
\end{gathered}
$$

where

$$
\mathbf{e}_{\mathrm{P}_{d}}=\left(\begin{array}{c}
\sin \theta_{d} \cos \phi_{d} \\
\sin \theta_{d} \sin \phi_{d} \\
\cos \theta_{d}
\end{array}\right),
$$

$$
\mathbf{e}_{\mathrm{SV}_{d}}=\left(\begin{array}{c}
\cos \theta_{d} \cos \phi_{d} \\
\cos \theta_{d} \sin \phi_{d} \\
-\sin \theta_{d}
\end{array}\right),
$$

and

$$
\mathbf{e}_{\mathrm{SH}_{d}}=\left(\begin{array}{c}
-\sin \phi_{d} \\
\cos \phi_{d} \\
0
\end{array}\right) .
$$

The subscripts $i$ and $d$ indicate incidence and diffraction, respectively. The two angles $\theta_{i}$ and $\phi_{i}$ represent incidence angles, and other two angles $\theta_{d}$ and $\phi_{d}$ correspond to diffraction angles as shown in Figure 1. We define $\mathbb{R}_{s}^{\mathrm{P}}, \mathbb{R}_{s}^{\mathrm{SV}}$, and $\mathbb{R}_{s}^{\mathrm{SH}}$ as source radiation patterns of incident $\mathrm{P}-, \mathrm{SV}$ - and $\mathrm{SH}$-waves from the seismic source $\mathbf{f}_{\mathrm{s}}$. The six terms $\mathbb{R}_{m, Q}^{\mathrm{P}-\mathrm{P}}, \mathbb{R}_{m, Q}^{\mathrm{P}-\mathrm{SV}}, \mathbb{R}_{m, Q}^{\mathrm{P}-\mathrm{SH}}, \mathbb{R}_{m, Q}^{\mathrm{SV}-\mathrm{SV}}, \mathbb{R}_{m, Q}^{\mathrm{SV}-\mathrm{SH}}$, and $\mathbb{R}_{m, Q}^{\mathrm{SH}-\mathrm{SH}}$ indicate parameter radiation patterns in different parameterizations $(Q)$ recently considered (Gholami et al., 2013a; Operto et al., 2013; Alkhalifah and Plessix, 2014; Kamath and Tsvankin, 2016; Oh and Alkhalifah, 2016). In this study, we ignore $\mathbb{R}_{m, Q}^{\mathrm{SV}-\mathrm{P}}, \mathbb{R}_{m, O}^{\mathrm{SH}-\mathrm{P}}$, and $\mathbb{R}_{m, Q}^{\mathrm{SH}-\mathrm{SV}}$ because $\mathbb{R}_{m, Q}^{\mathrm{SV}-\mathrm{P}}$ is the same as $\mathbb{R}_{m, Q}^{\mathrm{P}-\mathrm{SV}}$ and the influences of the other two radiation patterns $\left(\mathbb{R}_{m, Q}^{\mathrm{SH}-\mathrm{P}}\right.$ and $\left.\mathbb{R}_{m, Q}^{\mathrm{SH}-\mathrm{SV}}\right)$ are weak
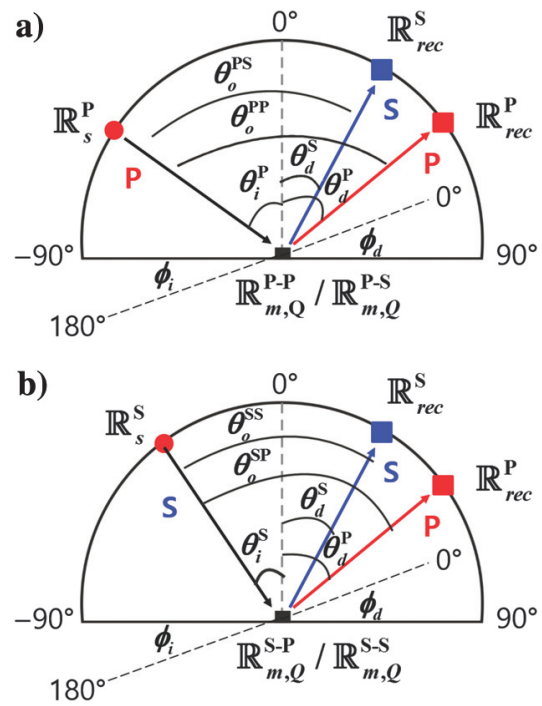

Figure 1. The schematic diagrams illustrating the mechanism of the total radiation response $\mathbb{R}_{\text {Total }}$ and the definition of the angles that are used throughout the paper: for incident (a) P-wave and (b) S-wave. The black, red, and blue arrows indicate the incident wave, reflected $\mathrm{P}$-wave, and reflected S-wave, respectively, which are propagated following Snell's law. The dashed lines denote the inline direction. The angles $\theta$ and $\varphi$ indicate the dip and azimuth angles. The superscripts indicate the types of body waves and subscripts, $i, d$, and $o$, mean the incidence, diffraction, and opening angles, respectively. The radiation patterns $\mathbb{R}_{s}, \mathbb{R}_{m}$, and $\mathbb{R}_{\text {rec }}$ indicate the source radiation, parameter radiation, and receiver patterns, respectively. 
in the conventional acquisition using an explosive source or a vertical vibroseis. For simplicity, we assume an isotropic background medium by using three orthonormal basis vectors $\left(\mathbf{e}_{\mathrm{P}}, \mathbf{e}_{\mathrm{SV}}\right.$, and $\mathbf{e}_{\mathrm{SH}}$ ), which indicate the particle motions of each wave in isotropic media so that we ignore the influence of anisotropy during wave propagation through the background medium. For this reason, this analysis is limited to the initial stage of the multiparameter FWI when the background medium is isotropic or weakly anisotropic.

The virtual source of a certain parameter $(m)$ as a point moment tensor source $\mathbf{M}_{m}$ (Wu and Aki, 1985; Jost and Herrmann, 1989) can be obtained by the chain rule (Kamath and Tsvankin, 2013; Bergslid et al., 2015) and the virtual sources of the elastic constants $\mathbf{M}_{C i j}$ (Oh and Alkhalifah, 2016) as follows:

$$
\mathbf{M}_{m}^{k_{i}, Q}=\sum_{i j} \frac{\partial C_{i j}^{Q}}{\partial p_{m, n}} \mathbf{M}_{C i j}^{k_{i}},
$$

where the superscript $k_{i}$ indicates the type of incident waves (P, SV, and $\mathrm{SH}$ ). In Appendix A, we show the moment tensor form of the nine elastic constants. Because each elastic constant $C_{i j}$ is defined by different parameters depending on the parameterization $(Q)$, the virtual source for a certain parameter in a certain parameterization has a different linear combination of the virtual sources for the elastic constants, which finally causes a different source mechanism of each parameter. The virtual source as a point source $\mathbf{f}_{m}$ from isotropic incident wavefields can be expressed as follows:

$$
\mathbf{f}_{m}^{k_{i}, Q}=\omega^{2} \frac{\partial \rho}{\partial p_{m, n}} \mathbf{e}_{k_{i}}
$$

The vectors $\mathbf{e}_{k_{i}}$ denote the orthonormal basis vectors for the incident waves along the directions of $\mathrm{P}-, \mathrm{SV}-$, and $\mathrm{SH}$-wave particle motions. The term $\omega^{2}$ originates from the time derivatives in equation 8 . From equations 16 and 17 , we notice that the point source of the virtual source $\mathbf{f}_{m}$ is only activated by the density perturbation, whereas the point moment tensor source of the virtual source $\mathbf{M}_{m}$ is activated by any parameter related to the elastic constants.

Finally, we define the total radiation responses of the PDW as follows:

$$
\mathbb{R}_{\operatorname{Total}(m, Q)}^{k_{i}-k_{d}}=\left|\mathbf{u}_{\operatorname{PDW}(m, Q)}^{k_{i}-k_{d}}\left(\theta_{i}, \phi_{i}, \theta_{d}, \phi_{d}\right)\right|=\mathbb{R}_{s}^{k_{i}} \mathbb{R}_{m, Q}^{k_{i}-k_{d}} \mathbb{R}_{\mathrm{rec}}^{k_{d}},
$$

where

$$
\mathbb{R}_{\text {rec }}^{k_{d}}=\left|\mathbf{e}_{k_{d}}\right|
$$

In the above equations, the total radiation response of the PDW $\mathbb{R}_{\text {Total }}$ consists of the source radiation pattern $\mathbb{R}_{s}$, the parameter radiation pattern $\mathbb{R}_{m}$, and the receiver radiation pattern $\mathbb{R}_{\text {rec }}$ (Figure 1). In this study, we only consider parameter radiation patterns assuming that the seismic source generates isotropic $\mathrm{P}$ - and $\mathrm{S}$-waves (although this is not realistic) and we record all three wavefield components.

\section{CONVENTIONAL PARAMETERIZATIONS FOR 3D ELASTIC ORTHORHOMBIC MEDIA}

As an initial step toward developing a practical orthorhombic elastic inversion strategy, we need to first study the sensitivity of the data to the parameterization of choice, which provides considerable insights into the potential tradeoff between the chosen parameters. Such a study for 3D elastic orthorhombic media requires an analytic study of the sensitivities of the parameters in terms of scattering angle and azimuth, as well as mode conversion in the data.

Before introducing the new parameterization, we discuss two possible conventional parameterizations (CPs) for 3D elastic orthorhombic media in this section. The first one includes eight seismic velocities, one Thomsen's parameter $\left(\delta_{3}\right)$ (Thomsen, 1986), and the density, which is called the conventional parameterization I (CP-I) throughout the paper. The second parameterization, which is called the conventional parameterization II (CP-II) includes two seismic velocities, seven Thomsen's parameters, and the density, which has been the preferred parameterization for surface seismic data for years (Tsvankin, 1997).

\section{CP-I: Eight seismic velocities plus one Thomsen's parameter}

The CP-I includes 10 independent parameters as follows:

$$
\left(\begin{array}{llllllllll}
V_{\mathrm{P} 1} & V_{\mathrm{P} 2} & V_{\mathrm{P} 3} & V_{n 1} & V_{n 2} & \delta_{3} & V_{\mathrm{S} 1} & V_{\mathrm{S} 2} & V_{\mathrm{S} 3} & \rho
\end{array}\right),
$$

where $V_{\mathrm{P} 1}, V_{\mathrm{P} 2}$, and $V_{\mathrm{P} 3}$ denote the $\mathrm{P}$-wave velocities in $x$-, $y$-, and $z$-directions, respectively, $V_{\mathrm{S} 1}$ and $V_{\mathrm{S} 2}$ indicate the vertical $\mathrm{S}$-wave velocities that are polarized in the $x z$ - and $y z$-planes, respectively, and $V_{\mathrm{S} 3}$ is the horizontal $\mathrm{S}$-wave velocity in the $x z$-plane but propagating in $x$-direction. The terms $V_{n 1}$ and $V_{n 2}$ are the normal moveout (NMO) P-wave velocities for horizontal reflectors in the $x z$ - and $y z$-planes, respectively, and $\delta_{3}$ is an anisotropic parameter in the $x y$-plane (Song and Alkhalifah, 2013). This is the orthorhombic version of the conventional 2D elastic VTI parameterization, which is suggested by Kamath and Tsvankin (2013). Although we use the seismic velocities rather than square velocities, this does not affect the shape of the radiation pattern for the parameters. Even though Kamath and Tsvankin (2013) analyze this parameterization for the transmission data, we will focus on surface seismic data by plotting the surface-to-surface (STS) radiation pattern (Oh and Alkhalifah, 2016) as expressed in Appendix B. To calculate the radiation patterns of the parameters in CP-I, we first express each elastic constant in terms of the CP-I parameters as follows:

$\left(\begin{array}{l}C_{11} \\ C_{22} \\ C_{33} \\ C_{12} \\ C_{13} \\ C_{23} \\ C_{44} \\ C_{55} \\ C_{66}\end{array}\right)=\left(\begin{array}{c}\rho V_{\mathrm{P} 1}^{2} \\ \rho V_{\mathrm{P} 2}^{2} \\ \rho V_{\mathrm{P} 3}^{2} \\ \rho\left[\sqrt{\left(V_{\mathrm{P} 1}^{2}-V_{\mathrm{S} 3}^{2}\right)\left(\left(1+2 \delta_{3}\right) V_{\mathrm{P} 1}^{2}-V_{\mathrm{S} 3}^{2}\right)}-V_{\mathrm{S} 3}^{2}\right] \\ \rho\left[\sqrt{\left(V_{\mathrm{P} 3}^{2}-V_{\mathrm{S} 1}^{2}\right)\left(V_{n 1}^{2}-V_{\mathrm{S} 1}^{2}\right)}-V_{\mathrm{S} 1}^{2}\right] \\ \rho\left[\sqrt{\left(V_{\mathrm{P} 3}^{2}-V_{\mathrm{S} 2}^{2}\right)\left(V_{n 2}^{2}-V_{\mathrm{S} 2}^{2}\right)}-V_{\mathrm{S} 2}^{2}\right] \\ \rho V_{s 2}^{2} \\ \rho V_{s 1}^{2} \\ \rho V_{s 3}^{2}\end{array}\right)$

With these equations, we obtain the moment tensor forms of the virtual sources for each CP-I parameter corresponding to an isotropic background model $V_{\mathrm{P}}, V_{\mathrm{S}}$, and $\rho$ ) using the chain rule in equation 16 
and the virtual sources of the elastic constants (Appendix A) as follows:

$$
\begin{aligned}
& \mathbf{M}_{V_{\mathrm{P} 1}}^{k_{i}, \mathrm{CP}-\mathrm{I}}=\frac{2 \rho V_{\mathrm{P}}}{h}\left(\begin{array}{ccc}
\varepsilon_{x x}^{k_{i}}+\varepsilon_{y y}^{k_{i}} & 0 & 0 \\
0 & \varepsilon_{x x}^{k_{i}} & 0 \\
0 & 0 & 0
\end{array}\right), \\
& \mathbf{M}_{V_{\mathrm{P} 2}}^{k_{i}, \mathrm{CP}-\mathrm{I}}=\frac{2 \rho V_{\mathrm{P}}}{h}\left(\begin{array}{ccc}
0 & 0 & 0 \\
0 & \varepsilon_{y y}^{k_{i}} & 0 \\
0 & 0 & 0
\end{array}\right), \\
& \mathbf{M}_{V_{\mathrm{P} 3}}^{k_{i}, \mathrm{CP}-\mathrm{I}}=\frac{\rho V_{\mathrm{P}}}{h}\left(\begin{array}{ccc}
\varepsilon_{z z}^{k_{i}} & 0 & 0 \\
0 & \varepsilon_{z z}^{k_{i}} & 0 \\
0 & 0 & \varepsilon_{z z}^{k_{i}}
\end{array}\right) \\
& +\frac{\rho V_{\mathrm{P}}\left(\varepsilon_{x x}^{k_{i}}+\varepsilon_{y y}^{k_{i}}+\varepsilon_{z z}^{k_{i}}\right)}{h}\left(\begin{array}{ccc}
0 & 0 & 0 \\
0 & 0 & 0 \\
0 & 0 & 1
\end{array}\right) \text {, } \\
& \mathbf{M}_{V_{n 1}}^{k_{i}, \mathrm{CP}-\mathrm{I}}=\frac{\rho V_{\mathrm{P}}}{h}\left(\begin{array}{ccc}
\varepsilon_{z z}^{k_{i}} & 0 & 0 \\
0 & 0 & 0 \\
0 & 0 & \varepsilon_{x x}^{k_{i}}
\end{array}\right) \\
& \mathbf{M}_{V_{n 2}}^{k_{i}, \text { CP-I }}=\frac{\rho V_{\mathrm{P}}}{h}\left(\begin{array}{ccc}
0 & 0 & 0 \\
0 & \varepsilon_{z z}^{k_{i}} & 0 \\
0 & 0 & \varepsilon_{y y}^{k_{i}}
\end{array}\right), \\
& \mathbf{M}_{\delta_{3}}^{k_{i}, \mathrm{CP}-\mathrm{I}}=\frac{\rho V_{\mathrm{P}}^{2}}{h}\left(\begin{array}{ccc}
\varepsilon_{y y}^{k_{i}} & 0 & 0 \\
0 & \varepsilon_{x x}^{k_{i}} & 0 \\
0 & 0 & 0
\end{array}\right), \\
& \mathbf{M}_{V_{\mathrm{S} 1}}^{k_{i}, \mathrm{CP}-\mathrm{I}}=\frac{4 \rho V_{\mathrm{S}}}{h}\left(\begin{array}{ccc}
-\varepsilon_{z z}^{k_{i}} & 0 & \varepsilon_{x z}^{k_{i}} \\
0 & 0 & 0 \\
\varepsilon_{x z}^{k_{i}} & 0 & -\varepsilon_{x x}^{k_{i}}
\end{array}\right), \\
& \mathbf{M}_{V_{\mathrm{S} 2}}^{k_{i}, \mathrm{CP}-\mathrm{I}}=\frac{4 \rho V_{\mathrm{S}}}{h}\left(\begin{array}{ccc}
0 & 0 & 0 \\
0 & -\varepsilon_{z z}^{k_{i}} & \varepsilon_{y z}^{k_{i}} \\
0 & \varepsilon_{y z}^{k_{i}} & -\varepsilon_{y y}^{k_{i}}
\end{array}\right), \\
& \mathbf{M}_{V_{\mathrm{S} 3}}^{k_{i}, \mathrm{CP}-\mathrm{I}}=\frac{4 \rho V_{\mathrm{S}}}{h}\left(\begin{array}{ccc}
-\varepsilon_{y y}^{k_{i}} & \varepsilon_{x y}^{k_{i}} & 0 \\
\varepsilon_{x y}^{k_{i}} & -\varepsilon_{x x}^{k_{i}} & 0 \\
0 & 0 & 0
\end{array}\right), \\
& \mathbf{f}_{\rho}^{k_{i}, \mathrm{CP}-\mathrm{I}}=\omega^{2} \mathbf{e}_{k_{i}},
\end{aligned}
$$

$$
\begin{aligned}
\mathbf{M}_{\rho}^{k_{i}, \mathrm{CP}-\mathrm{I}}= & \frac{V_{\mathrm{P}}^{2}\left(\varepsilon_{x x}^{k_{i}}+\varepsilon_{y y}^{k_{i}}+\varepsilon_{z z}^{k_{i}}\right)}{h}\left(\begin{array}{ccc}
1 & 0 & 0 \\
0 & 1 & 0 \\
0 & 0 & 1
\end{array}\right) \\
& +\frac{2 V_{\mathrm{S}}^{2}}{h}\left(\begin{array}{ccc}
-\varepsilon_{y y}^{k_{i}}-\varepsilon_{z z}^{k_{i}} & \varepsilon_{x y}^{k_{i}} & \varepsilon_{x z}^{k_{i}} \\
\varepsilon_{x y}^{k_{i}} & -\varepsilon_{z z}^{k_{i}}-\varepsilon_{x x}^{k_{i}} & \varepsilon_{y z}^{k_{i}} \\
\varepsilon_{x z}^{k_{i}} & \varepsilon_{y z}^{k_{i}} & -\varepsilon_{y y}^{k_{i}}-\varepsilon_{x x}^{k_{i}}
\end{array}\right),
\end{aligned}
$$

where $h$ is the grid size that will be used for FWI. Notice that the virtual source for the density has a point source (equation 22j) and a point moment tensor source (equation $22 \mathrm{k}$ ). Because we consider a point scatterer in FWI, the grid size indicates the length of scatterer and affects the scattering mechanism depending on the wavelength of incident wave (refer to Figure 2 in $\mathrm{Wu}$ and Aki, 1988). For the density perturbation, the scattering mechanism should be more complicated because the combination of $\mathbf{f}_{\rho}$ and $\mathbf{M}_{\rho}$ can be changed depending on the wavelength of incident wave ( $\omega$ in $\left.\mathbf{f}_{\rho}\right)$ and the size of the virtual source ( $h$ in $\mathbf{M}_{\rho}$ ) while other nine parameters have Rayleigh scattering if we use a small-enough grid for the FWI. Using these moment tensors, we obtain the total radiation response and the STS radiation pattern for each parameter.

First, we display the diffraction pattern that shows how much energy propagates through the medium along the reflection and transmission raypaths. The diffraction patterns for isotropic elastic parameters have been well-studied (Wu and Aki, 1985; Tarantola, 1986). However, anisotropic parameters have more complicated diffraction patterns depending on the incidence angle; thus, we consider diffraction patterns of each parameter at different incidence angles. To visualize the diffraction patterns of P-P- and P-SV-waves in equation 18, we calculate the PDW for each parameter through forward modeling (Graves, 1996). We place a single explosive source, which generates only incident P-waves, at the middle of the surface with $0.8 \mathrm{~km}$ depth (red circle). We perturb each parameter at 13 grid points (eight on the source and receiver plane, four at $45^{\circ}$ incidence angle in inline and crossline, and one at normal incidence) that are of equal distance from the source and of different incidence angles from the source (refer to Appendix $\mathrm{C}$ for more details).

In Figure 2, we display the diffraction patterns for each CP-I parameter. From Figures 3 to 7, we also display the STS radiation patterns assuming a horizontal reflector (refer to Appendix B). Here, we show how the moment tensor descriptions from equations 22 a to $22 \mathrm{k}$ can be connected to the diffraction pattern (Figure 2) and, finally, the STS radiation pattern (from Figures 3 to 7), which can be useful to interpret the tradeoffs between parameters. As we can see from moment tensor description of $V_{\mathrm{P} 3}$ perturbation in equation $22 \mathrm{c}$, $V_{\mathrm{P} 3}$ perturbation generates a vertically dominant explosive source because of the second term in equation 22c. For this reason, in the diffraction pattern (Figure 2a), we observe a strong P-P-wave at the normal incidence (red arrow). However, through the transmission raypath (yellow arrow), we observe that there are no lateral diffractions of P-P- and P-SV-waves, and thus, we cannot record laterally propagating $\mathrm{P}-\mathrm{P}$ - and $\mathrm{P}-\mathrm{SV}$-waves of the $\mathrm{PDW}$ for $V_{\mathrm{P} 3}$ perturbation on the surface. This is because, in equation $22 \mathrm{c}, V_{\mathrm{P} 3}$ perturbation loses its isotropic explosive source term, whose momentum is a vertical strain that equals to zero when the wave propagates laterally. For this reason, in the STS radiation pattern of $V_{\mathrm{P} 3}$ perturbation (Figure 3), we only observe strong energy for P-P-waves at narrow open- 
ing angle. As Alkhalifah (2015b) shows, the cosine of opening angle (so-called scattering angle) is proportional to the recovered model wavenumber of each model parameter. Therefore, for $V_{\mathrm{P} 3}$ in CP-I, we expect high-wavenumber updates from the reflection data. For P-SV-waves, we expect slightly lower wavenumber updates although they are not low enough due to the critical angle.

One interesting feature of the STS radiation pattern for $V_{\mathrm{P} 3}$ (Figure 3) is the lack of SV-SV-waves. This can be also explained by a moment tensor decomposition as shown in equation 22c. The virtual source for $V_{\mathrm{P} 3}$ consists of an explosive source, which does not generate diffracted S-wave motions, and a vertical normal force whose magnitude is the summation of all the normal strains that is equivalent with the divergence of incident wavefields. For this reason, using the surface acquired data, SV-SV-waves have minor influence on the FWI for $V_{\mathrm{P} 3}$.

In the same manner as $V_{\mathrm{P} 3}$, the behaviors of other parameters in CP-I can be interpreted. First, the radiation patterns for two horizontal P-wave velocities $\left(V_{\mathrm{P} 1}\right.$ and $\left.V_{\mathrm{P} 2}\right)$ show clear separations along the azimuth angles because they dominantly have normal forces acting along the horizontal direction as their moment tensors demonstrate in a)
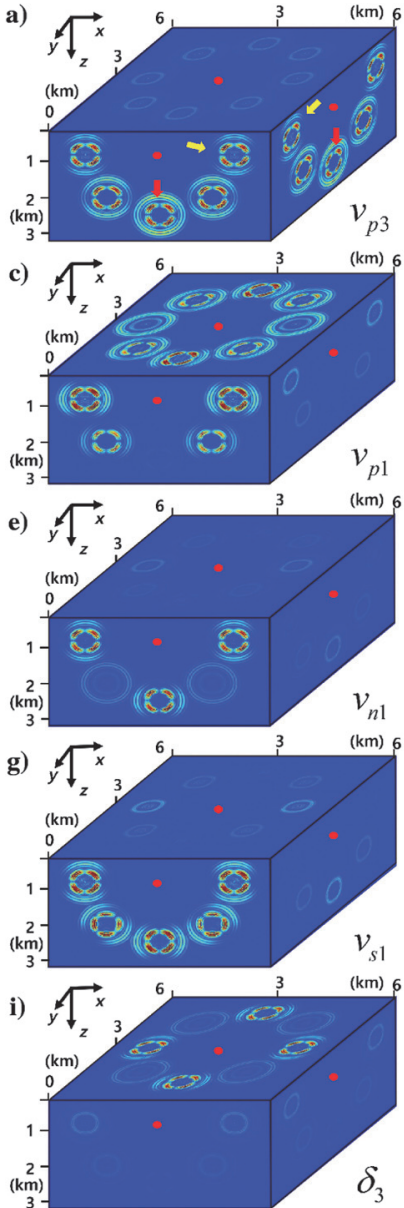

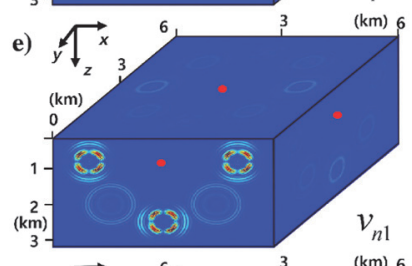

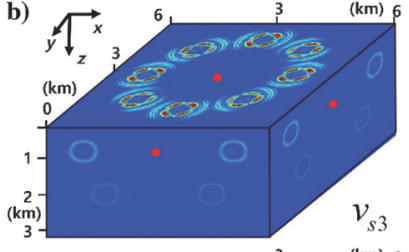

d)
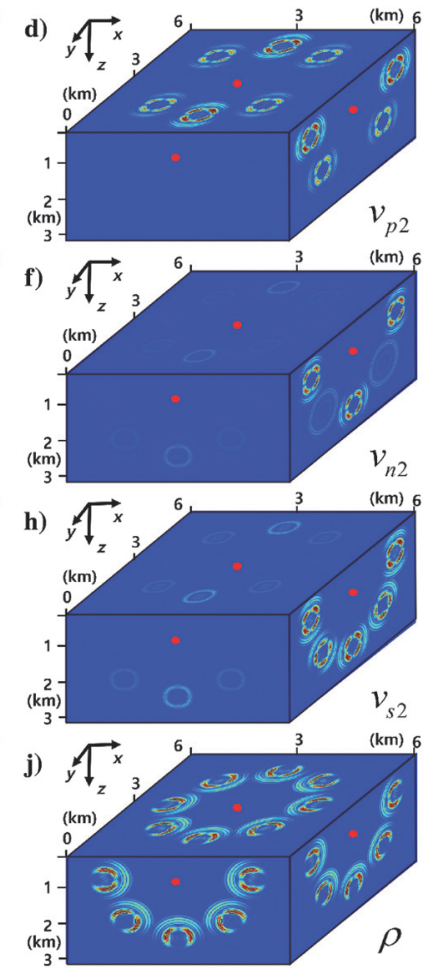

Figure 2. The snapshots of the PDW for the 10 independent parameters in CP-I. An explosive source is located at $(3,3,0.8 \mathrm{~km})$ (red circles at each plane). We assume 13 perturbations at the same time, which are far from the source with the same distance. The $x z$ $(y=3 \mathrm{~km}), y z-(x=3 \mathrm{~km})$, and $x y$-planes $(z=0.8 \mathrm{~km})$ contain the source position. Appendix $\mathrm{C}$ will be helpful when interpreting this figure. equations 22a and 22b. As a result, the virtual sources for two horizontal P-wave velocities (Figure $2 \mathrm{c}$ and $2 \mathrm{~d}$ ) diffract most of the energy horizontally, which allows us to recover the horizontal $\mathrm{P}$-wave velocities from the receivers with a wide opening angle (Figure 4). Therefore, we expect low-wavenumber updates for two horizontal P-wave velocities focusing on the shallower depth than $V_{\mathrm{P} 3}$.

For the three S-wave velocities (Figure $2 b, 2 g$, and $2 \mathrm{~h}$ ), the diffraction patterns show rotational behaviors around the source location. This is because, as demonstrated in equations $22 \mathrm{~g}, 22 \mathrm{~h}$, and $22 \mathrm{i}$, the additional implosive sources make the diffraction patterns rotate so that most of the energy is diffracted along the normal direction to the incidence waves. For $V_{\mathrm{S} 3}$, the rotation occurs on the $x y$-plane (Figure $2 \mathrm{~b}$ ). As a result, we observe that no $\mathrm{P}$ - or $\mathrm{S}$-wave energies are reflected and transmitted along the source-receiver line thus, for the horizontal reflector, we cannot recover $V_{\mathrm{S} 3}$ without the $\mathrm{SH}-\mathrm{SH}$-wave as shown in Figure 3. However, for two vertical Swave velocities $\left(V_{\mathrm{S} 1}\right.$ and $\left.V_{\mathrm{S} 2}\right)$, the rotation occurs around the $y$ and $x$-axes, respectively (Figure $2 \mathrm{~g}$ and $2 \mathrm{~h}$ ). As a result, when the incidence angle is $45^{\circ}$, the maximum energy of P-P-waves will be diffracted along the reflection raypath (Figure 5), whereas there will be no energy along the reflection path at $0^{\circ}$ incidence or the transmission path at $90^{\circ}$ incidence as shown in Figure $2 \mathrm{~g}$ ( $x z$-plane) and $2 \mathrm{~h}$ ( $y z$-plane).

The moment tensor forms of the two NMO velocities are equivalent to $C_{13}$ and $C_{23}$ (Oh and Alkhalifah, 2016). In elastic multiparameter FWI, focusing on the P-P-wave, these two parameters have strong tradeoffs with two vertical $\mathrm{S}$-wave velocities $\left(V_{\mathrm{S} 1}\right.$ and $V_{\mathrm{S} 2}$ ) as shown in Figures 5 and 6. From the moment tensor forms in equation $22 \mathrm{~d}, 22 \mathrm{e}, 22 \mathrm{~g}$, and $22 \mathrm{~h}$, we notice that, at $0^{\circ}$ and $90^{\circ}$ incidence angles, in which shear strains equal to zero, the source mechanisms for the vertical S-wave velocities are the

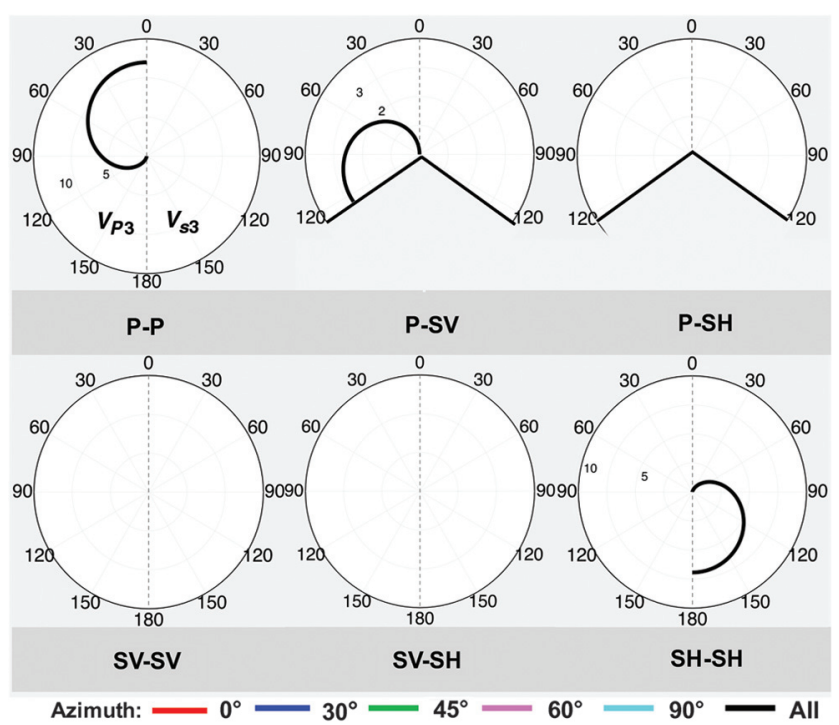

Figure 3. The STS radiation pattern for $V_{\mathrm{P} 3}$ (left hemisphere) and $V_{\mathrm{S} 3}$ (right hemisphere) perturbations in CP-I. The angle is defined as the opening angle. Notice that mode-converted P-S-waves have the opening angle narrower than $180^{\circ}$ due to the critical angle in equation B-4. We do not scale the radiation patterns of each parameter. The values inside each circle indicate relative magnitude of each radiation pattern per a unit perturbation. Poisson's ratio is assumed to be 0.25 . 
same as those for the NMO velocities. Even at a $45^{\circ}$ incidence angle, because the NMO velocities have isotropic diffraction patterns on each plane, the influence of reflection data on FWI of the vertical S-wave and NMO velocities might be similar. In other words, the vertical S-wave and NMO velocities have strong tradeoffs for STS acquired data although their source mechanisms are different. However, from Figures 5 and 6 , we notice that these tradeoffs can be partially mitigated if credible P-SV-, SV-SV-, and SV-SH-waves are acquired.

The $\delta_{3}$ can only be recovered by receivers at intermediate azimuth angles with wide opening angle as the diffraction pattern (Figure $2 \mathrm{i}$ ) and the STS radiation pattern (Figure 7) show, which might be the weakest area in a 3D narrow azimuth survey. The density perturbation (Figure $2 \mathrm{j}$ ) shows a somewhat unique diffraction pattern compared with other parameters, in which most energy is back scattered. This is because a point source in equation $22 \mathrm{j}$ generates unidirectional particle motions (the time derivative of the wavefields does not change the propagation direction), whereas a point moment tensor source in equation $22 \mathrm{k}$ generates bidirectional particle motions (the divergence of the wavefields is equivalent to the stress). For this reason, the forward scattering is cancelled out, but the backward scattering is enhanced. This cancellation can occur only in the back scattering area if we change the parameterization from velocity type to the impedance type (Operto et al., 2013; Ramos-Martinez et al., 2016).

As the STS radiation patterns illustrate, the parameters in CP-I show good separation along the azimuth angles. However, if we look through the STS radiation patterns for all the parameters (Figures 3 7), we notice that the radiation patterns for CP-I are very complicated because most parameters generate all kinds of possible reflected waves. Moreover, SH-waves are not helpful to reduce the tradeoff between two corresponding parameters, $\left[V_{\mathrm{P} 1}\right.$ and $\left.V_{\mathrm{P} 2}\right],\left[V_{n 1}\right.$ and $\left.V_{n 2}\right]$, and $\left[V_{\mathrm{S} 1}\right.$ and $\left.V_{\mathrm{S} 2}\right]$, along the $x$ - and $y$-axes because all the $\mathrm{SH}$-waves have the same STS radiation pattern in both parameters. In addition, as shown in Figure 3, this parameterization might fail to

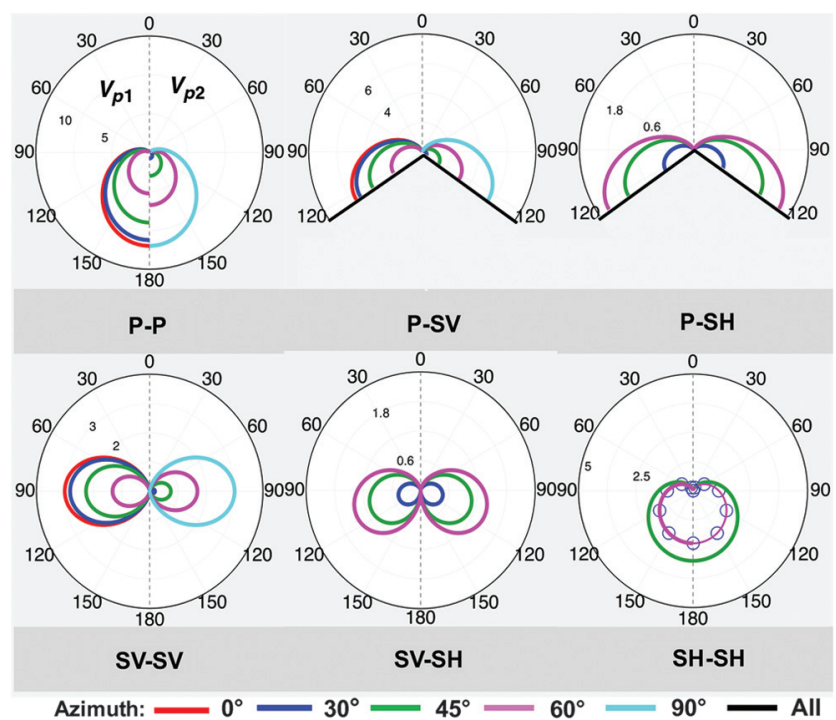

Figure 4 . The same as Figure 3 but for $V_{\mathrm{P} 1}$ (left hemisphere) and $V_{\mathrm{P} 2}$ (right hemisphere) perturbations in CP-I. When the radiation pattern has same amplitude at $30^{\circ}$ and $60^{\circ}$ azimuth angles, blue open circles are used instead of blue solid line. recover $V_{\mathrm{S} 3}$ related to $C_{66}$, which affects the kinematics of the Swave propagation. Accordingly, in CP-I, two vertical S-wave velocities, $V_{\mathrm{S} 1}$ and $V_{\mathrm{S} 2}$, are updated from the data with the intermediate scattering angle, whereas $V_{\mathrm{S} 3}$ is not updated significantly. For this reason, after FWI without any special constraint, we always obtain elastic orthorhombic models regardless of the subsurface properties although there should be still many isotropic and VTI rocks in real earth. Even for $\left[V_{\mathrm{P} 1}, V_{\mathrm{P} 2}, V_{\mathrm{S} 3}\right]$, this parameterization is not promising when wide azimuth data and good isotropic initial models are unavailable. This is because $V_{\mathrm{S} 3}$ is updated with high resolution, whereas $V_{\mathrm{P} 1}$ and $V_{\mathrm{P} 2}$ are updated with low resolution, but from narrow and wide azimuth data, respectively. In conclusion, if we do not

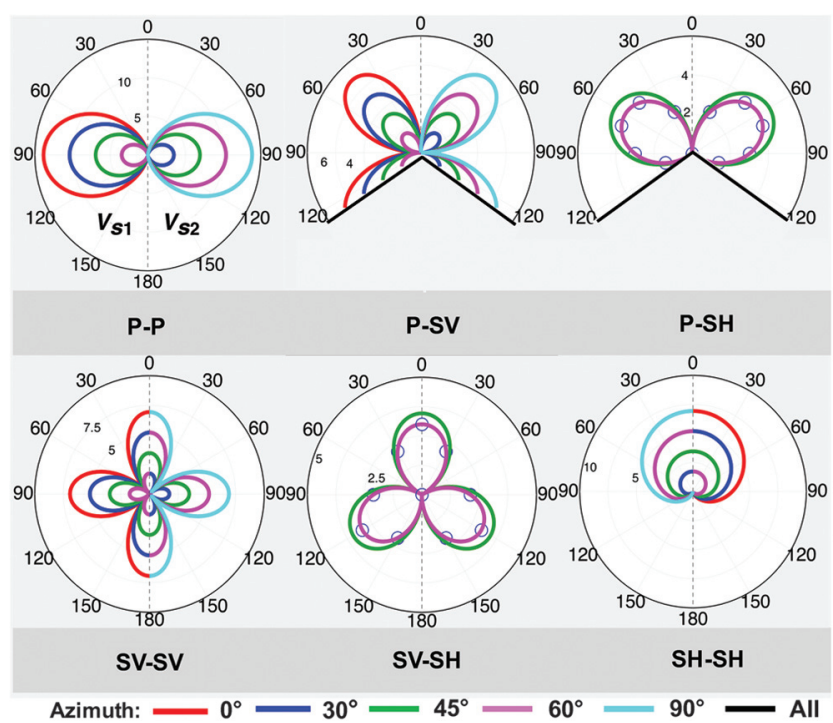

Figure 5. The same as Figure 3 but for $V_{\mathrm{S} 1}$ (left hemisphere) and $V_{\mathrm{S} 2}$ (right hemisphere) perturbations in CP-I.

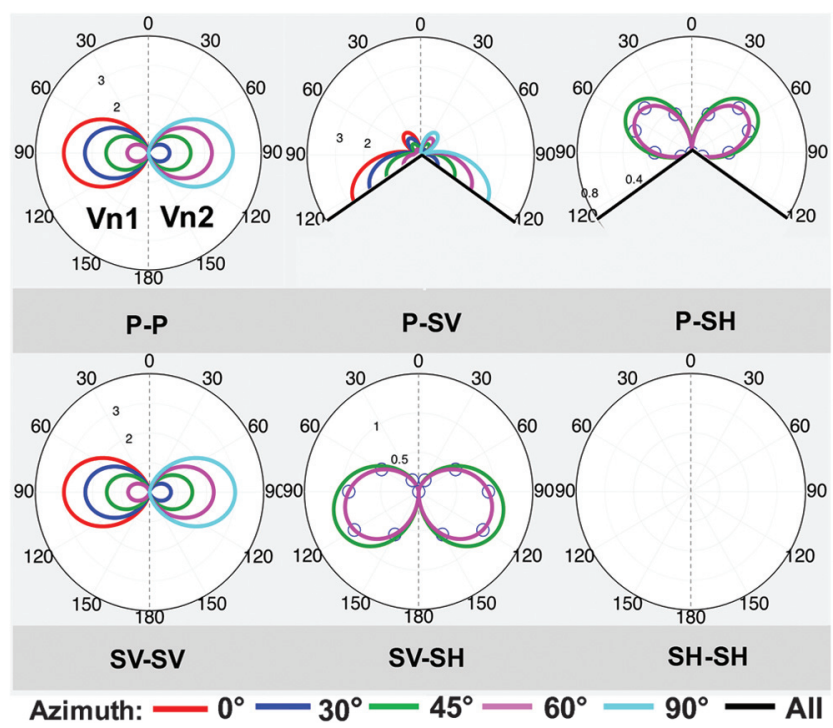

Figure 6. The same as Figure 3 but for $V_{n 1}$ (left hemisphere) and $V_{n 2}$ (right hemisphere) perturbations in CP-I. 
start from a good isotropic or VTI initial model, the multiparameter FWI using CP-I might not be promising.

\section{CP-II: Two velocities plus seven Thomsen's parameters}

The CP-II includes two seismic velocities, seven Thomsen's parameters, and density as follows:

$$
\left(\begin{array}{llllllllll}
V_{\mathrm{P} 3} & \varepsilon_{1} & \varepsilon_{2} & \delta_{1} & \delta_{2} & \delta_{3} & V_{\mathrm{S} 3} & \gamma_{1} & \gamma_{2} & \rho
\end{array}\right),
$$

where $\varepsilon_{1}$ and $\varepsilon_{2}$ denote the VTI parameter $\varepsilon$ in the $x z$ - and $y z$-planes, respectively. The parameters $\delta_{1}$ and $\delta_{2}$ indicate the VTI parameter $\delta$ in the $x z$ - and $y z$-planes, and $\gamma_{2}$ and $\gamma_{1}$ are the VTI parameter $\gamma$ in the $x z$ - and $y z$-planes (Tsvankin, 1997), respectively. Using CP-II, the elastic constants can be expressed as follows:

$$
\left(\begin{array}{l}
C_{11} \\
C_{22} \\
C_{33} \\
C_{12} \\
C_{13} \\
C_{23} \\
C_{44} \\
C_{55} \\
C_{66}
\end{array}\right)=\left(\begin{array}{c}
\left(1+2 \varepsilon_{1}\right) \rho V_{\mathrm{P} 3}^{2} \\
\left(1+2 \varepsilon_{2}\right) \rho V_{\mathrm{P} 3}^{2} \\
\rho V_{\mathrm{P} 3}^{2} \\
\rho\left[\sqrt{\left(\left(1+2 \varepsilon_{1}\right) V_{\mathrm{P} 3}^{2}-V_{\mathrm{S} 3}^{2}\right)\left(\left(1+2 \delta_{3}\right)\left(1+2 \varepsilon_{1}\right) V_{\mathrm{P} 3}^{2}-V_{\mathrm{S} 3}^{2}\right)}-V_{\mathrm{S} 3}^{2}\right] \\
\rho\left[\sqrt{\left(V_{\mathrm{P} 3}^{2}-\frac{V_{\mathrm{S} 3}^{2}}{1+2 \gamma_{1}}\right)\left(\left(1+2 \delta_{1}\right) V_{\mathrm{P} 3}^{2}-\frac{V_{\mathrm{S} 3}^{2}}{1+2 \gamma_{1}}\right)}-\frac{V_{\mathrm{S} 3}^{2}}{1+2 \gamma_{1}}\right] \\
\rho\left[\sqrt{\left(V_{\mathrm{P} 3}^{2}-\frac{V_{\mathrm{S} 3}^{2}}{1+2 \gamma_{2}}\right)\left(\left(1+2 \delta_{2}\right) V_{\mathrm{P} 3}^{2}-\frac{V_{\mathrm{S} 3}^{2}}{1+2 \gamma_{2}}\right)}-\frac{V_{\mathrm{S} 3}^{2}}{1+2 \gamma_{2}}\right] \\
\frac{\rho V_{\mathrm{S} 3}^{2}}{1+2 \gamma_{2}} \\
\frac{\rho V_{\mathrm{S}}^{2}}{1+2 \gamma_{1}} \\
\rho V_{\mathrm{S} 3}^{2}
\end{array}\right)
$$

In the same manner as what we did for CP-I, we obtain the moment tensor form of the virtual sources for CP-II parameters in the isotropic background model as follows:

$$
\begin{gathered}
\mathbf{M}_{V_{\mathrm{P} 3}}^{k_{i}, \mathrm{CP}-\mathrm{II}}=\frac{2 \rho V_{\mathrm{P}}\left(\varepsilon_{x x}^{k_{i}}+\varepsilon_{y y}^{k_{i}}+\varepsilon_{z z}^{k_{i}}\right)}{h}\left(\begin{array}{lll}
1 & 0 & 0 \\
0 & 1 & 0 \\
0 & 0 & 1
\end{array}\right), \\
\mathbf{M}_{\varepsilon_{1}}^{k_{i}, \mathrm{CP}-\mathrm{II}}=\frac{2 \rho V_{\mathrm{P}}^{2}}{h}\left(\begin{array}{ccc}
\varepsilon_{x x}^{k_{i}}+\varepsilon_{y y}^{k_{i}} & 0 & 0 \\
0 & \varepsilon_{x x}^{k_{i}} & 0 \\
0 & 0 & 0
\end{array}\right), \\
\mathbf{M}_{\varepsilon_{2}}^{k_{i}, \mathrm{CP}-\mathrm{II}}=\frac{2 \rho V_{\mathrm{P}}^{2}}{h}\left(\begin{array}{ccc}
0 & 0 & 0 \\
0 & \varepsilon_{y y}^{k_{i}} & 0 \\
0 & 0 & 0
\end{array}\right), \\
\mathbf{M}_{\delta_{1}^{k_{i}}, \mathrm{CP}-\mathrm{II}}=\frac{\rho V_{\mathrm{P}}^{2}}{h}\left(\begin{array}{ccc}
\varepsilon_{z z}^{k_{i}} & 0 & 0 \\
0 & 0 & 0 \\
0 & 0 & \varepsilon_{x x}^{k_{i}}
\end{array}\right), \\
\mathbf{M}_{\delta_{2}}^{k_{i}, \mathrm{CP}-\mathrm{II}}=\frac{\rho V_{\mathrm{P}}^{2}}{h}\left(\begin{array}{ccc}
0 & 0 & 0 \\
0 & \varepsilon_{z z}^{k_{i}} & 0 \\
0 & 0 & \varepsilon_{y y}^{k_{i}}
\end{array}\right),
\end{gathered}
$$

$$
\mathbf{M}_{\delta_{3}}^{k_{i}, \mathrm{CP}-\mathrm{II}}=\frac{\rho V_{\mathrm{P}}^{2}}{h}\left(\begin{array}{ccc}
\varepsilon_{y y}^{k_{i}} & 0 & 0 \\
0 & \varepsilon_{x x}^{k_{i}} & 0 \\
0 & 0 & 0
\end{array}\right),
$$

$$
\mathbf{M}_{V_{\mathrm{S} 3}}^{k_{i}, \mathrm{CP}-\mathrm{II}}=\frac{4 \rho V_{\mathrm{S}}}{h}\left(\begin{array}{ccc}
-\varepsilon_{z z}^{k_{i}}-\varepsilon_{y y}^{k_{i}} & \varepsilon_{x y}^{k_{i}} & \varepsilon_{x z}^{k_{i}} \\
\varepsilon_{x y}^{k_{i}} & -\varepsilon_{z z}^{k_{i}}-\varepsilon_{x x}^{k_{i}} & \varepsilon_{y z}^{k_{i}} \\
\varepsilon_{x z}^{k_{i}} & \varepsilon_{y z}^{k_{i}} & -\varepsilon_{y y}^{k_{i}}-\varepsilon_{x x}^{k_{i}}
\end{array}\right),
$$

$$
\mathbf{M}_{\gamma_{1}}^{k_{i}, \mathrm{CP}-\mathrm{II}}=\frac{4 \rho V_{\mathrm{S}}}{h}\left(\begin{array}{ccc}
\varepsilon_{z z}^{k_{i}} & 0 & -\varepsilon_{x z}^{k_{i}} \\
0 & 0 & 0 \\
-\varepsilon_{x z}^{k_{i}} & 0 & \varepsilon_{x x}^{k_{i}}
\end{array}\right),
$$

$$
\begin{aligned}
& \mathbf{M}_{\gamma_{2}}^{k_{i}, \mathrm{CP}-\mathrm{II}}=\frac{4 \rho V_{\mathrm{S}}}{h}\left(\begin{array}{ccc}
0 & 0 & 0 \\
0 & \varepsilon_{z z}^{k_{i}} & -\varepsilon_{y z}^{k_{i}} \\
0 & -\varepsilon_{y z}^{k_{i}} & \varepsilon_{y y}^{k_{i}}
\end{array}\right), \\
& \mathbf{f}_{\rho}^{k_{i}, \text { CP-II }}=\omega^{2} \mathbf{e}_{k_{i}}, \\
& \mathbf{M}_{\rho}^{k_{i}, \mathrm{CP}-\mathrm{II}}=\frac{V_{\mathrm{P}}^{2}\left(\varepsilon_{x x}^{k_{i}}+\varepsilon_{y y}^{k_{i}}+\varepsilon_{z z}^{k_{i}}\right)}{h}\left(\begin{array}{ccc}
1 & 0 & 0 \\
0 & 1 & 0 \\
0 & 0 & 1
\end{array}\right) \\
& +\frac{2 V_{\mathrm{S}}^{2}}{h}\left(\begin{array}{ccc}
-\varepsilon_{y y}^{k_{i}}-\varepsilon_{z z}^{k_{i}} & \varepsilon_{x y}^{k_{i}} & \varepsilon_{x z}^{k_{i}} \\
\varepsilon_{x y}^{k_{i}} & -\varepsilon_{z z}^{k_{i}}-\varepsilon_{x x}^{k_{i}} & \varepsilon_{y z}^{k_{i}} \\
\varepsilon_{x z}^{k_{i}} & \varepsilon_{y z}^{k_{i}} & -\varepsilon_{y y}^{k_{i}}-\varepsilon_{x x}^{k_{i}}
\end{array}\right) \text {. }
\end{aligned}
$$

Notice that the virtual sources for $\delta_{3}$ and $\rho$ are the same as in CP-I.

In Figure 8, we display the diffraction patterns for each CP-II parameter we obtain from the same background model with that for CP-I. From equations 25a and $25 \mathrm{~g}$, we notice that the virtual sources for $V_{\mathrm{P} 3}$ and $V_{\mathrm{S} 3}$ are the same as the virtual sources for the $\mathrm{P}$ - and $\mathrm{S}$-wave velocities in isotropic elastic media (refer to Appendix B in Oh and Alkhalifah, 2016). The vertical P-wave velocity $V_{\mathrm{P} 3}$ generates an isotropic explosion, whose momentum is the divergence of the incident wavefields, so that the incident $\mathrm{S}$-waves are cancelled out. For this reason, as Figure 8a shows, the P-wave velocity only generates P-P-waves of the PDW in isotropic background media (Wu and Aki, 1985; Tarantola, 1986). The virtual source for the $\mathrm{S}$-wave velocity in equation $25 \mathrm{~g}$ can be decomposed into three double-coupled forces and one implosion-type force. As a result, the radiation pattern for the $\mathrm{S}$-wave velocity is rotated on the source location as Figure $8 \mathrm{~b}$ shows. For this reason, using CP-II might be a better choice than CP-I when we do not start FWI from a good isotropic initial model. No matter which direction of P- and $\mathrm{S}$-waves we choose, they should have the same radiation patterns as those of the isotropic elastic parameterization in an isotropic background model if we use dimensionless parameters to define other velocities. However, the choice of the direction for seismic velocities affects the radiation pattern for the remaining dimensionless parameters (Alkhalifah, 2016). In Figures 9, 10, 11, and 12, we display the STS radiation pattern for CP-II parameters. We do not display the 
radiation pattern for $\delta_{3}$ and the density because their radiation patterns are the same as those in CP-I (Figure 7).

First, if we only consider P-and $\mathrm{SV}$-waves, $\varepsilon_{1}$ and $\varepsilon_{2}$ have their maxima at wide opening angles, whereas $\delta_{1}$ and $\delta_{2}$ have their maximum at intermediate opening angle. We observe that the $\gamma$ parameters also have their maxima at intermediate opening angles, which means that there might be strong tradeoffs between $\delta$ and $\gamma$, although P-SVand SV-SV-waves for $\gamma$ have their maxima at narrow and wide opening angles. These observations indicate that there are strong tradeoffs between Thomsen's parameters at intermediate opening angles, which prevents us from recovering the subsurface anisotropic features. The choice of the vertical P-wave velocity, as we will see next, causes these strong tradeoffs between anisotropic parameters. In addition, we cannot effectively constrain the long wavelength vertical $\mathrm{P}$-wave velocity from surface seismic data. Therefore, using the horizontal P-wave velocity is a better choice to reduce tradeoffs between anisotropic parameters as Alkhalifah and Plessix (2014) mention.

With respect to the azimuth direction, we notice that the diffraction patterns for each dimensionless parameter group are well-separated along the azimuth direction (also shown in Figure 8). This azimuthal separation of the radiation pattern might be good to distinguish $\varepsilon_{1}$ from $\varepsilon_{2}$, as well as $\delta_{1}$ from $\delta_{2}$ and $\gamma_{1}$ from $\gamma_{2}$. However, if we do not have wide enough azimuth data or good VTI initial models, CP-II might not be promising in the same logic for CP-I because we cannot invert for $\varepsilon_{2}, \delta_{2}$, and $\gamma_{2}$, which should be the same as $\varepsilon_{1}, \delta_{1}$, and $\gamma_{1}$ for the subsurface VTI properties, without wide azimuth data. We also expect that this parameterization might be highly sensitive to the accurate description of the azimuth of the orthorhombic model (so-called tilted orthorhombic media).

\section{NEW PARAMETERIZATION FOR 3D ELASTIC ORTHORHOMBIC MEDIA}

\section{New parameterization with deviation parameters}

As shown in the previous section "Conventional parameterizations for 3D elastic orthorhombic media," the parameters in CP-I have quite complicated radiation patterns that are caused by complex mode conversions and strong trade-offs between parameters. On the other hand, the parameters in CP-II have clear decoupling of isotropy from anisotropic characteristics while there are still some complexities caused by coupling of VTI and HTI properties.

To overcome these limitations, we suggest a new elastic orthorhombic parameterization that has outstanding separation of anisotropic characteristics based on the parameter class as follows:

$$
\left(\begin{array}{llllllllll}
V_{\mathrm{P} 1} & \varepsilon_{1} & \varepsilon_{D} & \eta_{1} & \eta_{D} & \delta_{3} & V_{\mathrm{S} 1} & \gamma_{1} & \gamma_{D} & \rho
\end{array}\right)
$$

where

$$
\begin{aligned}
& \varepsilon_{D}=\frac{\varepsilon_{2}-\varepsilon_{1}}{1+2 \varepsilon_{1}}, \\
& \eta_{D}=\frac{\eta_{2}-\eta_{1}}{1+2 \eta_{1}},
\end{aligned}
$$

and

$$
\gamma_{D}=\frac{\gamma_{1}-\gamma_{2}}{1+2 \gamma_{2}}
$$

The new parameterization is built based on the acoustic orthorhombic parameterization suggested by Masmoudi and Alkhalifah (2016). We also use the horizontal P-wave velocity $V_{\mathrm{P} 1}$ as the

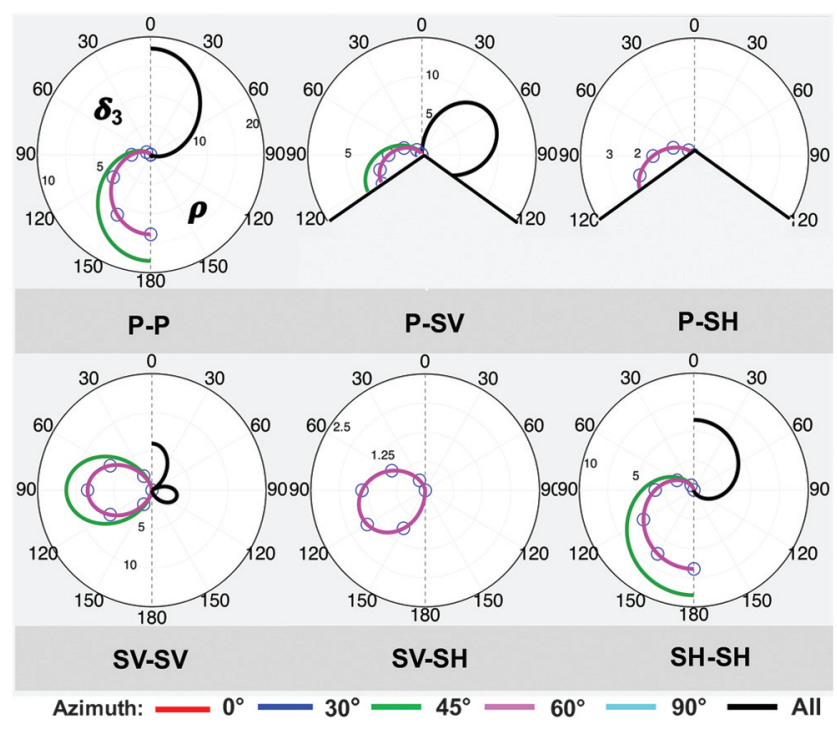

Figure 7. The same as Figure 3 but for $\delta_{3}$ (left hemisphere) and the density (right hemisphere) perturbations in CP-I, CP-II, and the new parameterization. Notice that these parameters have identical radiation patterns in all three parameterizations in this paper. a)
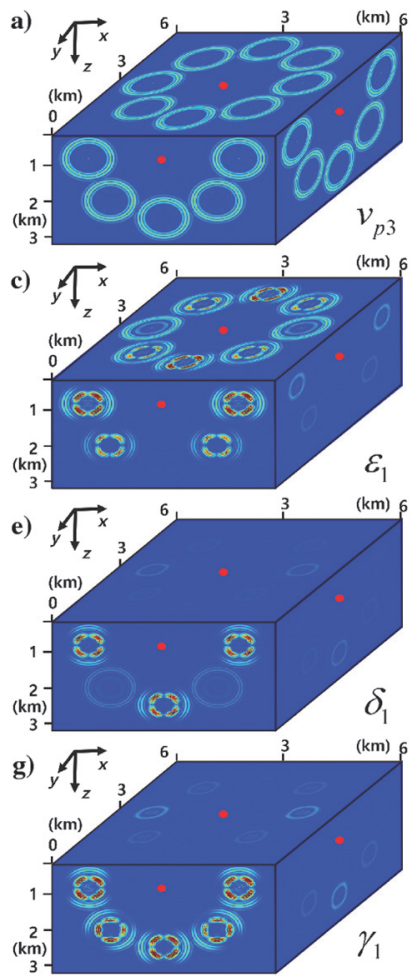
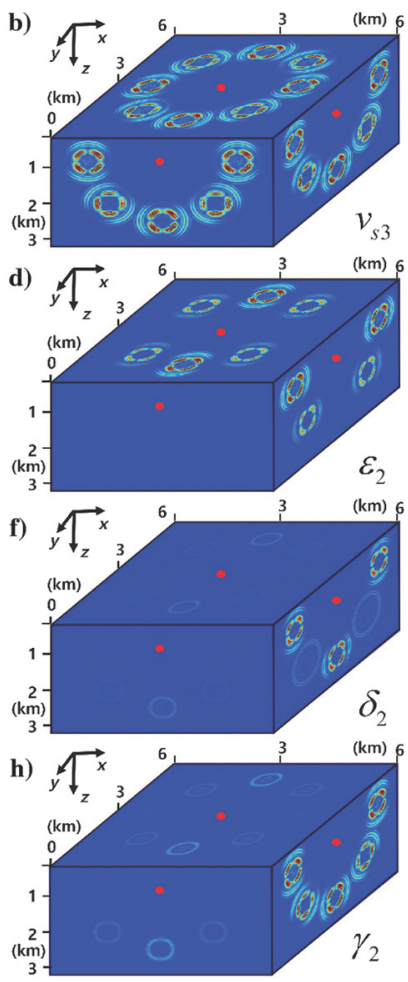

Figure 8. The snapshots of the PDW for the eight independent parameters in CP-II excluding $\delta_{3}$ and $\rho$, which have the same patterns with those in CP-I. The numerical settings are the same as Figure C1. 
central parameter and describe the polar variations using three VTI parameters $\left(\varepsilon_{1}, \eta_{1}\right.$, and $\left.\gamma_{1}\right)$. Compared to CP-II, we use $\eta_{1}$ $\left(V_{n 1}=V_{\mathrm{P} 3} \sqrt{1+2 \delta_{1}}\right.$ and $\left.V_{\mathrm{P} 1}=V_{n 1} \sqrt{1+2 \eta_{1}}\right)$, which is introduced by Alkhalifah (2003). We describe the azimuth variation using $\delta_{3}$ and three deviation parameters, $\varepsilon_{D}$ and $\eta_{D}$ from Masmoudi and Alkhalifah (2016) and $\gamma_{D}$ from Tsvankin (1997). Using the new parameterization, the elastic constants can be expressed as follows:
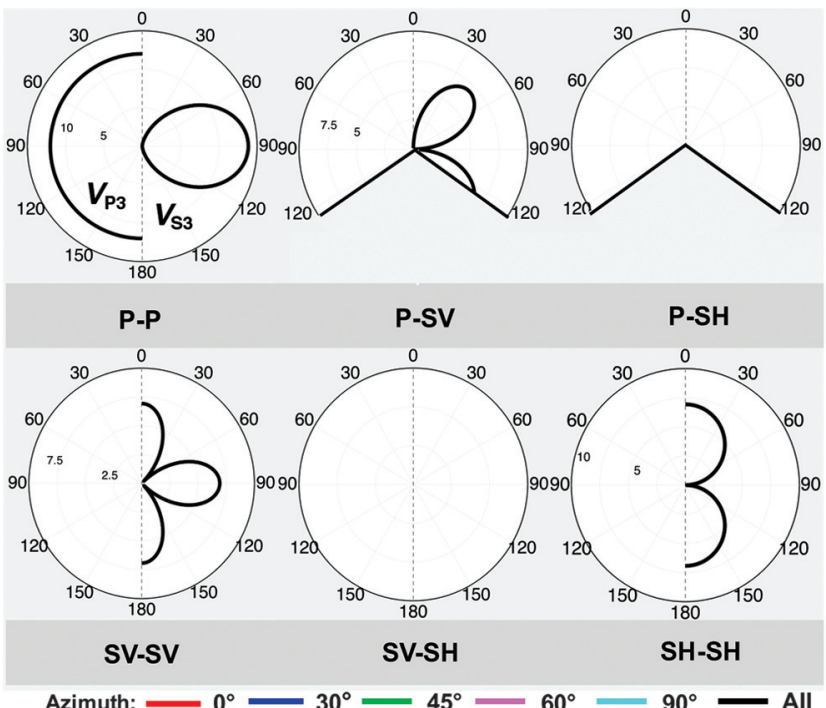

Figure 9. The STS radiation pattern for $V_{\mathrm{P} 3}$ (left hemisphere) and $V_{\mathrm{S} 3}$ (right hemisphere) perturbations in CP-II. The angle is defined as the opening angle. Notice that mode-converted P-S-waves have the opening angle narrower than $180^{\circ}$ due to the critical angle in equation B-4. Poisson's ratio is assumed to be 0.25 .
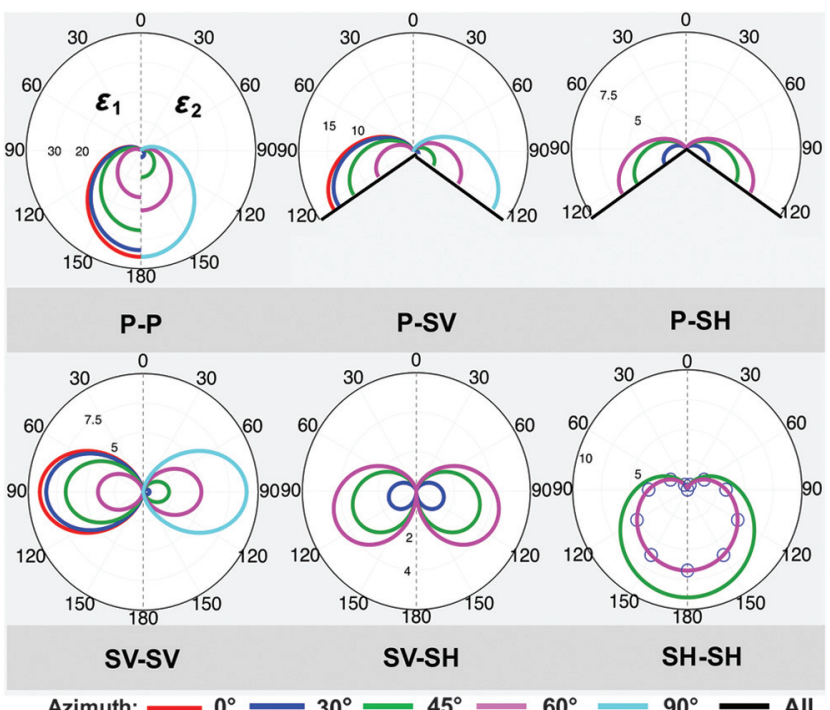

Figure 10. The same as Figure 9 but for $\varepsilon_{1}$ (left hemisphere) and $\varepsilon_{2}$ (right hemisphere) perturbations in CP-II.

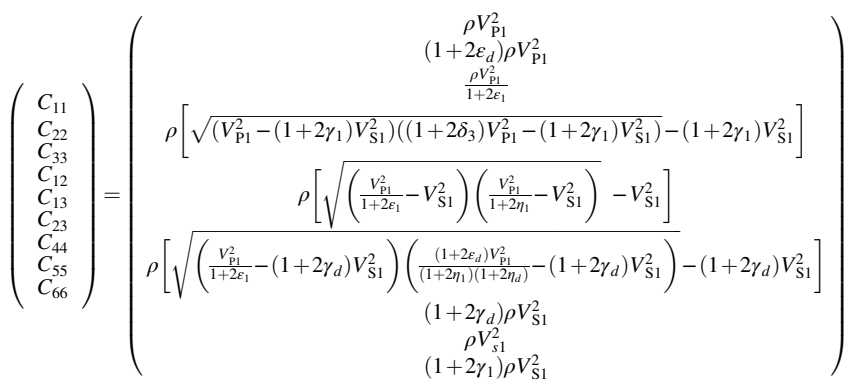

In the same manner as the CPs, we obtain the moment tensor form of the virtual sources for the new parameterization in the isotropic background model as follows:

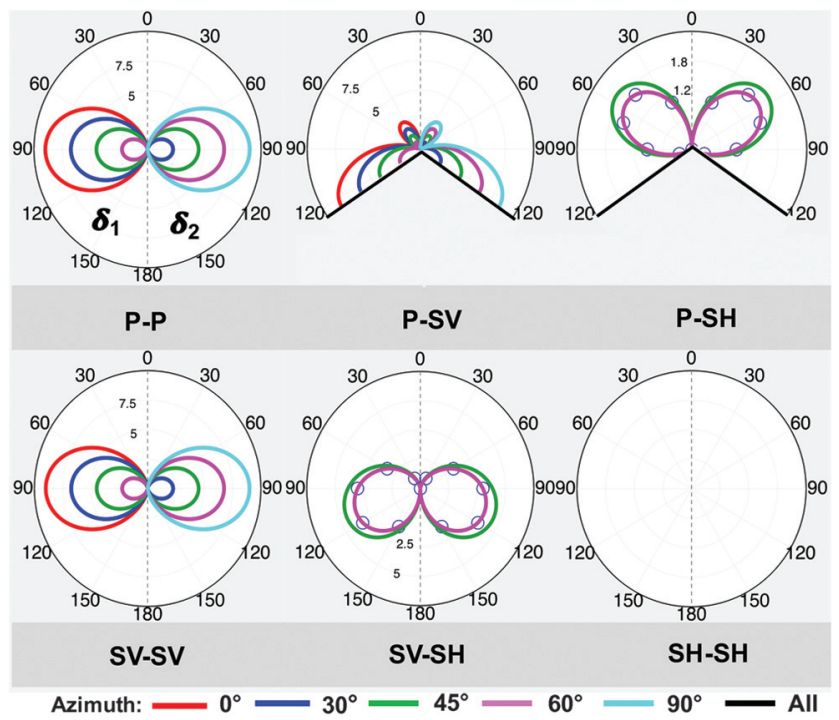

Figure 11. The same as Figure 9 but for $\delta_{1}$ (left hemisphere) and $\delta_{2}$ (right hemisphere) perturbations in CP-II.

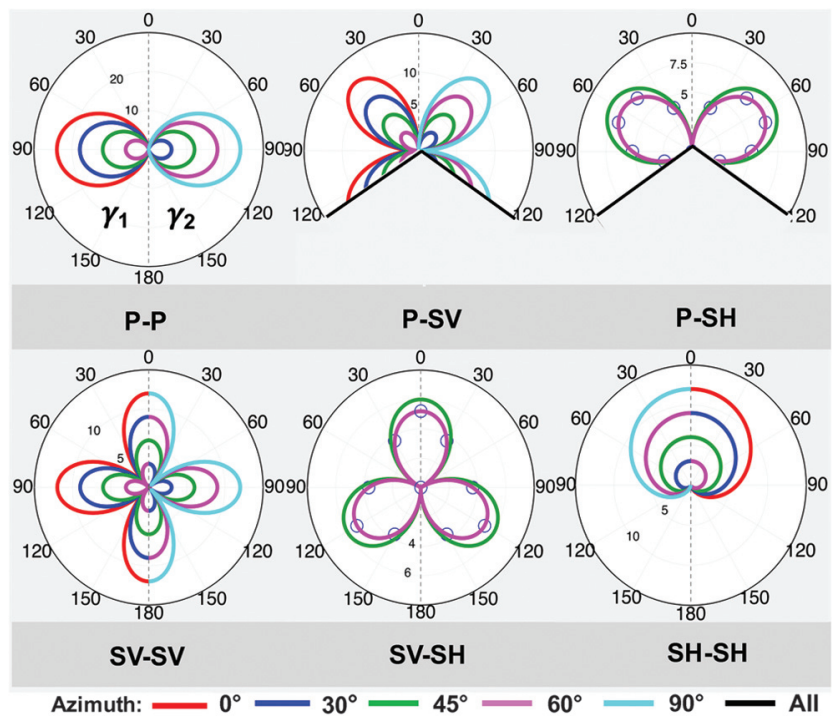

Figure 12. The same as Figure 9 but for $\gamma_{1}$ (left hemisphere) and $\gamma_{2}$ (right hemisphere) perturbations in CP-II. 


$$
\begin{aligned}
& \mathbf{M}_{V_{\mathrm{P} 1}}^{k_{i}, \mathrm{New}}=\frac{2 \rho V_{\mathrm{P}}\left(\varepsilon_{x x}^{k_{i}}+\varepsilon_{y y}^{k_{i}}+\varepsilon_{z z}^{k_{i}}\right)}{h}\left(\begin{array}{lll}
1 & 0 & 0 \\
0 & 1 & 0 \\
0 & 0 & 1
\end{array}\right), \\
& \mathbf{M}_{\varepsilon_{1}}^{k_{i} \text { New }}=\frac{-\rho V_{\mathrm{P}}^{2}}{h}\left(\begin{array}{ccc}
\varepsilon_{z z}^{k_{i}} & 0 & 0 \\
0 & \varepsilon_{z z}^{k_{i}} & 0 \\
0 & 0 & \varepsilon_{z z}^{k_{i}}
\end{array}\right) \\
& -\frac{-\rho V_{\mathrm{P}}^{2}\left(\varepsilon_{x x}^{k_{i}}+\varepsilon_{y y}^{k_{i}}+\varepsilon_{z z}^{k_{i}}\right)}{h}\left(\begin{array}{ccc}
0 & 0 & 0 \\
0 & 0 & 0 \\
0 & 0 & 1
\end{array}\right) \text {, } \\
& \mathbf{M}_{\varepsilon_{D}}^{k_{i} \text {,New }}=\frac{\rho V_{\mathrm{P}}^{2}}{h}\left(\begin{array}{ccc}
0 & 0 & 0 \\
0 & 2 \varepsilon_{y y}^{k_{i}}+\varepsilon_{z z}^{k_{i}} & 0 \\
0 & 0 & \varepsilon_{y y}^{k_{i}}
\end{array}\right), \\
& \mathbf{M}_{\eta_{1}}^{k_{i} \text { New }}=\frac{-\rho V_{\mathrm{P}}^{2}}{h}\left(\begin{array}{ccc}
\varepsilon_{z z}^{k_{i}} & 0 & 0 \\
0 & \varepsilon_{z z}^{k_{i}} & 0 \\
0 & 0 & \varepsilon_{x x}^{k_{i}}+\varepsilon_{y y}^{k_{i}}
\end{array}\right), \\
& \mathbf{M}_{\eta_{D}}^{k_{i} \text { New }}=\frac{-\rho V_{\mathrm{P}}^{2}}{h}\left(\begin{array}{ccc}
0 & 0 & 0 \\
0 & \varepsilon_{z z}^{k_{i}} & 0 \\
0 & 0 & \varepsilon_{y y}^{k_{i}}
\end{array}\right), \\
& \mathbf{M}_{\delta_{3}}^{k_{i}, \text { New }}=\frac{\rho V_{\mathrm{P}}^{2}}{h}\left(\begin{array}{ccc}
\varepsilon_{y y}^{k_{i}} & 0 & 0 \\
0 & \varepsilon_{x x}^{k_{i}} & 0 \\
0 & 0 & 0
\end{array}\right),
\end{aligned}
$$$$
\mathbf{M}_{V_{\mathrm{S} 1}}^{k_{i} \text { New }}=\frac{4 \rho V_{\mathrm{S}}}{h}\left(\begin{array}{ccc}
-\varepsilon_{z z}^{k_{i}}-\varepsilon_{y y}^{k_{i}} & \varepsilon_{x y}^{k_{i}} & \varepsilon_{x z}^{k_{i}} \\
\varepsilon_{x y}^{k_{i}} & -\varepsilon_{z z}^{k_{i}}-\varepsilon_{x x}^{k_{i}} & \varepsilon_{y z}^{k_{i}} \\
\varepsilon_{x z}^{k_{i}} & \varepsilon_{y z}^{k_{i}} & -\varepsilon_{y y}^{k_{i}}-\varepsilon_{x x}^{k_{i}}
\end{array}\right),
$$

$$
\mathbf{M}_{\gamma_{1}}^{k_{i} \text {,New }}=\frac{4 \rho V_{\mathrm{S}}}{h}\left(\begin{array}{ccc}
-\varepsilon_{y y}^{k_{i}} & \varepsilon_{x y}^{k_{i}} & 0 \\
\varepsilon_{x y}^{k_{i}} & -\varepsilon_{x x}^{k_{i}} & 0 \\
0 & 0 & 0
\end{array}\right)
$$$$
\mathbf{M}_{\gamma_{D}}^{k_{i} \text { New }}=\frac{4 \rho V_{\mathrm{S}}}{h}\left(\begin{array}{ccc}
0 & 0 & 0 \\
0 & \varepsilon_{z z}^{k_{i}} & -\varepsilon_{y z}^{k_{i}} \\
0 & -\varepsilon_{y z}^{k_{i}} & \varepsilon_{y y}^{k_{i}}
\end{array}\right),
$$$$
\mathbf{f}_{\rho}^{k_{i} \text { New }}=\omega^{2} \mathbf{e}_{k_{i}},
$$

$$
\begin{aligned}
\mathbf{M}_{\rho}^{k_{i}, \text { New }}= & \frac{V_{\mathrm{P}}^{2}\left(\varepsilon_{x x}^{k_{i}}+\varepsilon_{y y}^{k_{i}}+\varepsilon_{z z}^{k_{i}}\right)}{h}\left(\begin{array}{ccc}
1 & 0 & 0 \\
0 & 1 & 0 \\
0 & 0 & 1
\end{array}\right) \\
& +\frac{2 V_{\mathrm{S}}^{2}}{h}\left(\begin{array}{ccc}
-\varepsilon_{y y}^{k_{i}}-\varepsilon_{z z}^{k_{i}} & \varepsilon_{x y}^{k_{i}} & \varepsilon_{x z}^{k_{i}} \\
\varepsilon_{x y}^{k_{i}} & -\varepsilon_{z z}^{k_{i}}-\varepsilon_{x x}^{k_{i}} & \varepsilon_{y z}^{k_{i}} \\
\varepsilon_{x z}^{k_{i}} & \varepsilon_{y z}^{k_{i}} & -\varepsilon_{y y}^{k_{i}}-\varepsilon_{x x}^{k_{i}}
\end{array}\right) .
\end{aligned}
$$

Notice that the virtual source for $\delta_{3}$ and the density are the same as those in CP-I.

Figure 13 shows the diffraction patterns for each parameter and the STS radiation patterns are displayed in Figures 14, 15, 16, and 17. By choosing one P-wave velocity, one S-wave velocity, and seven dimensionless parameters, we also obtain isotropic radiation patterns for both seismic wavespeeds as equations 31a and $31 \mathrm{~g}$ demonstrate (Figure 13). However, the choice of the direction for both seismic velocities is an important issue because it affects the radiation patterns for other dimensionless parameters. As Alkhalifah (2016) reports, choosing the horizontal P-wave velocity is preferred because the radiation patterns for $\varepsilon$ move to narrow incidence angles (Figure 15), in which the density perturbation (Figure 7) also has its maximum scattering energy, so that the influence of the density can be absorbed by $\varepsilon$. Between the two horizontal P-wave velocities, we choose the $\mathrm{P}$-wave velocity along $x$-axis $\left(V_{\mathrm{P} 1}\right)$ because we prefer to make the radiation patterns for $\varepsilon_{D}$ (Figure 15) and $\eta_{D}$ (Figure 16) have their maxima at wide azimuth angles so that we recover the additional HTI parameters from the wide azimuth data.

The choice of the direction for the S-wave velocity also affects the radiation patterns for $\gamma$ parameters. Regarding the $S$-wave velocity, we choose the vertical S-wave velocity because, in this case, the influence of one of the $\gamma$ parameters on the STS acquired data can be mitigated (Figure 17) compared to CP-II. Between the two vertical $\mathrm{S}$-wave velocities, we choose the vertical $\mathrm{S}$-wave velocity polarized in $x z$-plane $\left(V_{\mathrm{S} 1}\right)$ for coincidence with the $\mathrm{P}$-wave motion we chose so that the radiation patterns for $\gamma_{D}$ also have their maximum at a
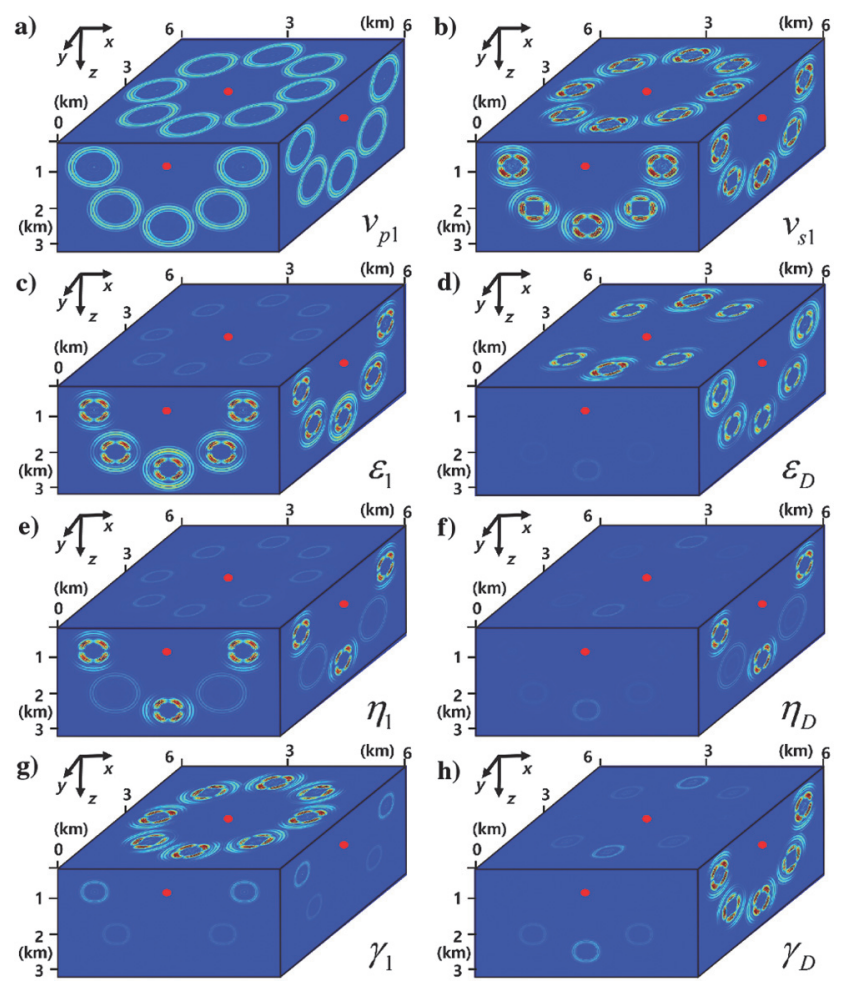

Figure 13. The snapshots of the PDW for the eight independent parameters in the new parameterization excluding $\delta_{3}$ and $\rho$, which have the same patterns as those in CP-I. The numerical settings are the same as Figure $\mathrm{C} 1$. 
wide azimuth angle (Figure 17). This allows us to invert for the isotropic $\left(V_{\mathrm{P} 1}\right.$ and $\left.V_{\mathrm{S} 1}\right)$ and VTI parameters $\left(\varepsilon_{1}\right.$ and $\left.\eta_{1}\right)$ with limited tradeoff in the narrow azimuth part of the data and the deviation parameters $\left(\varepsilon_{D}, \eta_{D}\right.$, and $\left.\gamma_{D}\right)$ can be recovered from the wide azimuth part.

\section{Characteristics of the new parameterization}

As we observed in the two CPs, the radiation patterns for many orthorhombic parameters are very complicated because most parameters generate all kinds of diffracted waves and there are lots

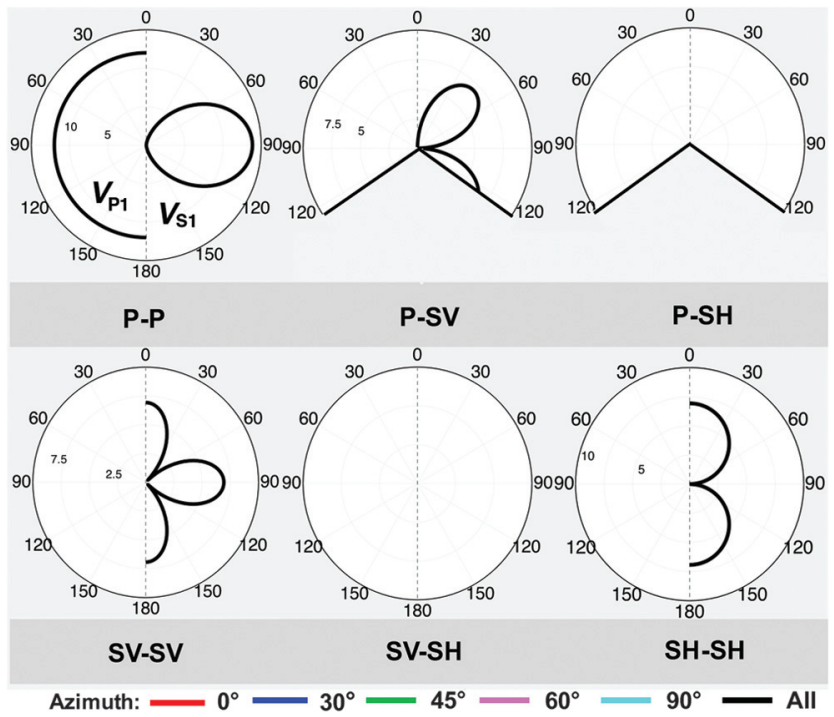

Figure 14. The STS radiation pattern for $V_{\mathrm{P} 1}$ (left hemisphere) and $V_{\mathrm{S} 1}$ (right hemisphere) perturbations in the new parameterization. The angle is defined as the opening angle. Notice that mode-converted P-S-waves have the opening angle narrower than $180^{\circ}$ due to the critical angle in equations B-4. Poisson's ratio is assumed to be 0.25 .

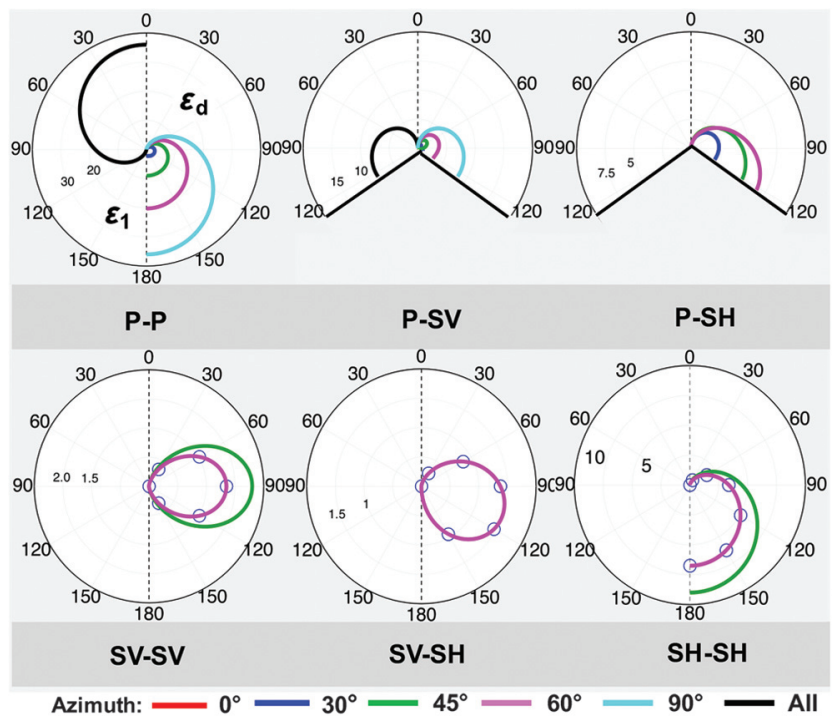

Figure 15. The same as Figure 14 but for $\varepsilon_{1}$ (left hemisphere) and $\varepsilon_{D}$ (right hemisphere) perturbations in the new parameterization. of tradeoffs among the 10 independent parameters. However, if we look through the STS radiation patterns of the new parameterization in Figure 7 and from Figures 14-17, we notice that the radiation patterns are well-separated in the new parameterization. The influences of the anisotropy and elasticity are decomposed in the new parameterization. The 10 independent parameters in the new parameterization can be divided into three groups: isotropic parameters $\left[V_{\mathrm{P} 1}, V_{\mathrm{S} 1}, \rho\right]$, VTI parameters $\left[\varepsilon_{1}, \eta_{1}, \gamma_{1}\right]$, and orthorhombic parameters for additional HTI characteristics $\left[\delta_{3}, \varepsilon_{D}, \eta_{D}, \gamma_{D}\right]$. We also note that we can separate the acoustic set (Masmoudi and Alkhalifah, 2016) to focus on P-P-waves, from the elastic description

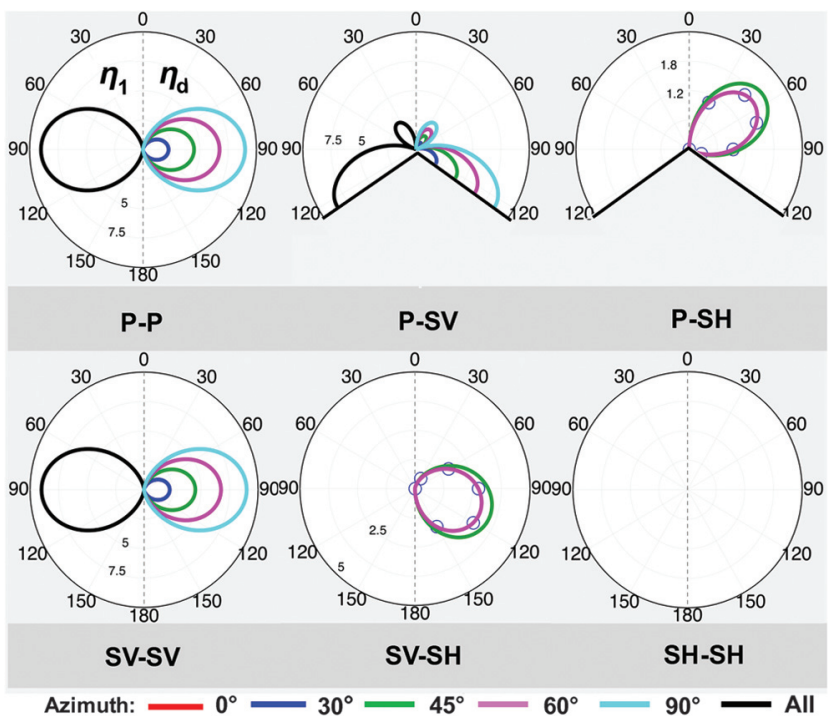

Figure 16. The same as Figure 14 but for $\eta_{1}$ (left hemisphere) and $\eta_{D}$ (right hemisphere) perturbations in the new parameterization.

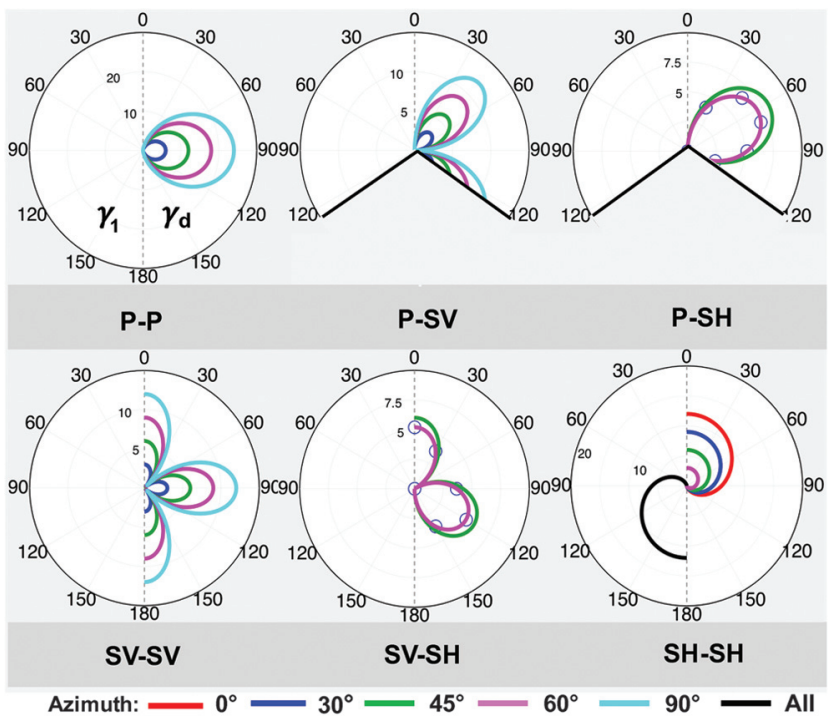

Figure 17. The same as Figure 14 but for $\gamma_{1}$ (left hemisphere) and $\gamma_{D}$ (right hemisphere) perturbations in the new parameterization. 
given by the additional parameters $\left[V_{\mathrm{S} 1}, \gamma_{1}, \gamma_{D}\right]$. Thus, in another continuation, we can update the elastic parameters when $\mathrm{S}$-wave information is available.

\section{DISCUSSION}

Throughout the paper, we focused on the moment tensor description, diffraction patterns, and STS radiation patterns of three parameterizations to determine which parameterization is optimal for 3D elastic orthorhombic FWI using the STS seismic data. We do not expect that we can invert for all 10 independent parameters using only STS data because, as we showed in the diffraction patterns (Figures 2, 8, and 13), we can record only a small portion of the diffraction energy on the surface seismic survey. Some parameters do not generate reliable $\mathrm{P}$ - and $\mathrm{SV}$-waves along the reflection and transmission raypaths so that we do not expect to recover these parameters from the earth's surface. The parameter $V_{\mathrm{S} 3}$ in CP-I is such a parameter that we cannot invert for without credible SH-SH-waves (Figure 3). Therefore, even though some structures are purely isotropic such as a salt body, CP-I will fail to recover this characteristic due to the lack of reliable $V_{\mathrm{S} 3}$ updates.

The pairs of parameters on $x z$ - and $y z$-planes, such as $\left[V_{\mathrm{P} 1}, V_{\mathrm{P} 2}\right]$, $\left[V_{n 1}, V_{n 2}\right]$, and $\left[V_{\mathrm{S} 1}, V_{\mathrm{S} 2}\right]$ in CP-I and $\left[\varepsilon_{1}, \varepsilon_{2}\right],\left[\delta_{1}, \delta_{2}\right]$, and $\left[\gamma_{1}, \gamma_{2}\right]$ in $\mathrm{CP}$-II, show good separations along the azimuth direction, which mean that these parameters can be recovered with fewer tradeoffs if wide azimuth data were available. However, we might not invert for the parameters on $y z$-plane if wide azimuth data were unavailable, which results in a failure to obtain the subsurface VTI properties. For this reason, when we do not start from a good VTI initial model, these two CPs do not look practical.

On the other hand, in the new parameterization, we can invert for 10 independent parameters in a different way because, as the STS radiation patterns suggest, the new parameterization has a good separation of the physics depending on the parameter. Using this characteristic, we can make the parameter updates be isotropic, VTI, or orthorhombic or even we can locally make the model parameter updates be isotropic, VTI, or orthorhombic by freezing the gradient directions of certain parameters at certain areas.

In addition, it seems that we cannot obtain some parameters in the new parameterization due to the lack of energy or strong tradeoffs between parameters, which allows us to reduce the number of invertible parameters. Where reliable $\mathrm{SH}-\mathrm{SH}$-wave and reliable wide azimuth data are not available, we can ignore $\gamma_{1}$ (Figure 17) and $\delta_{3}$ (Figure 7). Moreover, if we do not expect to have credible P-SV-, SV$\mathrm{SV}$-, and some $\mathrm{SH}$-waves such as monocomponent (vertical) OBC data, we cannot trust the inverted density because of the strong tradeoff with $\varepsilon_{1}$. In this case, we also cannot distinguish $\eta_{1}, \eta_{D}$, and $\gamma_{D}$ from the $S$-wave velocity because their STS radiation patterns for the P-P-wave have strong tradeoffs. Therefore, we do not expect to get reliable updates for $\eta_{1}, \eta_{D}$, and $\gamma_{D}$ until the $S$-wave velocity converges to its true value. However, in the multiparameter FWI for real data, it is hard to judge whether a certain parameter converged or not. In addition, these parameters have their maximum scattering at $90^{\circ}$ opening angles, which makes these parameters hard to be recovered (Alkhalifah, 2016). Therefore, in this case, we might need to ignore $\eta_{1}, \eta_{D}$, and $\gamma_{D}$ to increase the efficiency of the FWI algorithm.

\section{CONCLUSIONS}

We compare two CPs for 3D elastic orthorhombic media in terms of the parameter radiation patterns. We analyze diffraction patterns and STS radiation patterns to reveal the limitations of the CPs. Choosing one P-wave velocity, one S-wave velocity, seven dimensionless parameters, and density might be a reasonable choice because this makes both seismic velocities have isotropic behavior so that we can focus on the isotropic parameters in the early stages of FWI. However, for a better hierarchical feature, we suggest a new parameterization, which includes $V_{\mathrm{P} 1}, V_{\mathrm{S} 1}, \varepsilon_{1}, \eta_{1}$, $\gamma_{1}, \delta_{3}, \varepsilon_{D}, \eta_{D}, \gamma_{D}$, and $\rho$. The choice of the direction of P- and Swave velocities is important because it affects the radiation patterns of the other dimensionless parameters. We choose the horizontal P-wave velocity and vertical S-wave velocity polarized in the $x z$-plane. The choice of horizontal P-wave velocity makes the radiation pattern for $\varepsilon$ to focus on the narrow opening angle, which provides high resolution and less tradeoffs with $\eta$. The choice of the vertical S-wave velocity enables us ignore one of the two $\gamma$ parameters because this parameter diffracts most of the energy out of the source-receiver plane. We adopt the three deviation parameters because the choice of deviation parameters does not alter the radiation patterns of VTI parameters, which are azimuth independent. The radiation patterns for the new parameterization show reasonable hierarchical features in terms of the degree of anisotropy and participation of seismic waves.

\section{ACKNOWLEDGMENTS}

Research reported in this publication was supported by competitive research funding from King Abdullah University of Science and Technology (KAUST). For computer time, this research used the resources of the Supercomputing Laboratory at King Abdullah University of Science \& Technology (KAUST) in Thuwal, Saudi Arabia. We would like to thank the reviewer D. Köhn and three anonymous reviewers as well as the editors J. Shragge and S. Operto for their fruitful and constructive comments to improve the paper. We also thank the members of Seismic Wave Analysis Group in KAUST for the helpful discussions. Particularly, we would like to thank N. Masmoudi in KAUST for fruitful comments about the deviation parameters and V. Kazei at KAUST for finding some mathematical errors.

\section{APPENDIX A}

\section{REVIEW OF THE MOMENT TENSOR DESCRIP- TION OF THE VIRTUAL SOURCE}

We briefly review the previous study (Oh and Alkhalifah, 2016) on the moment tensor description of the virtual source for elastic constants as a function of the incidence angle. The virtual source of elastic constants as a point moment tensor source, which is the first virtual source in equation 8 , can be expressed as follows:

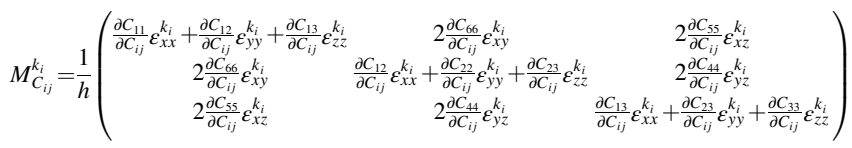

where $h$ is the grid size that will be used for FWI. The superscript $k_{i}$ indicates the types of waves (P, SV, and $\mathrm{SH})$. The amplitude varia- 
tion of strains as a function of incident angles can be approximated as follows (Kamath and Tsvankin, 2016):

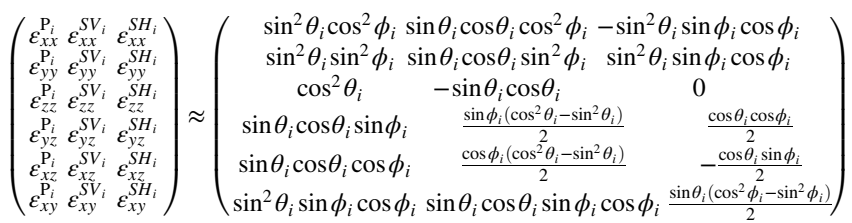

Here, subscript $i$ means the incident wave; thus, the two angles $\theta_{i}$ and $\phi_{i}$ indicate incidence angles as shown in Figure 1.

\section{APPENDIX B}

\section{STS RADIATION PATTERN}

For a better understanding of the radiation patterns of the PDW in anisotropic media, as Oh and Alkhalifah (2016) mention, we need to consider an incidence angle, as well as the diffraction angle. However, this approach is very complicated in a $3 \mathrm{D}$ problem because, as shown in equation 9, we need to consider four angles $\left(\theta_{i}, \phi_{i}, \theta_{d}\right.$, and $\left.\phi_{d}\right)$ at the same time. For this reason, we reformulate the radiation pattern as a function of the opening angle by assuming STS data acquisition and by considering Snell's law as previous works have done (Alkhalifah and Plessix, 2014; Oh and Alkhalifah, 2016). By assuming a horizontal reflector and the Snell's law (Figure 1), we can calculate the STS radiation pattern for each parameter, in which the angles are defined as opening angles as follows (Oh and Alkhalifah, 2016):

$$
\theta_{o}^{\mathrm{PP}}=\theta_{d}^{\mathrm{P}}-\theta_{i}^{\mathrm{P}},
$$

where

$$
\theta_{d}^{\mathrm{P}}=-\theta_{i}^{\mathrm{P}}
$$

and

$$
\theta_{o}^{\mathrm{PS}}=\theta_{d}^{\mathrm{S}}-\theta_{i}^{\mathrm{P}}
$$

where

$$
\theta_{d}^{\mathrm{S}}=\sin ^{-1}\left(\frac{V_{\mathrm{S}}}{V_{\mathrm{P}}} \sin \left(-\theta_{i}^{\mathrm{P}}\right)\right),
$$

where $V_{\mathrm{P}}$ and $V_{\mathrm{S}}$ denote the $\mathrm{P}$ - and $\mathrm{S}$-wave velocities, respectively.

\section{APPENDIX C RADIATION PATTERN OF $V_{P 3}$ IN CP-I}

In Appendix $\mathrm{C}$, as an example, we show how the radiation pattern of the PDW for a $V_{\mathrm{P} 3}$ perturbation can be obtained in the spherical coordinate system and how the radiation pattern can be used to interpret the complex behaviors of the PDW for the $V_{\mathrm{P} 3}$ perturbation. If we assume an isotropic source and $3 \mathrm{C}$ geophones in the isotropic homogeneous background media, the P-P-radiation pattern of the PDW in equation 9 for the $V_{\mathrm{P} 3}$ perturbation can be defined as follows:

$$
\mathbb{R}_{V_{\mathrm{P} 3}, \mathrm{CP}-\mathrm{I}}^{\mathrm{P}-\mathrm{P}}\left(\theta_{i}, \phi_{i}, \theta_{d}, \phi_{d}\right)=\mathbf{e}_{P_{d}}^{T} \mathbf{M}_{V_{\mathrm{P} 3}}^{P_{i}, \mathrm{CP}-\mathrm{I}} \mathbf{e}_{P_{d}} .
$$

If we plug the moment tensor of the $V_{\mathrm{P} 3}$ perturbation in equation $22 \mathrm{c}$ and the orthonormal basis function along the ray direction ( $\mathrm{P}$-wave particle motion) in equation 15 a into equation $\mathrm{C}-1$, we obtain equation C-2:

$$
\begin{aligned}
& \mathbb{R}_{V_{\mathrm{P} 3}, \mathrm{CP}-\mathrm{I}}^{\mathrm{P}-\mathrm{I}}\left(\theta_{i}, \phi_{i}, \theta_{d}, \phi_{d}\right)=\frac{\rho V_{\mathrm{P}}}{h}\left(\begin{array}{c}
\sin \theta_{d} \cos \phi_{d} \\
\sin \theta_{d} \sin \phi_{d} \\
\cos \theta_{d}
\end{array}\right)^{T} \\
& \quad \times\left(\begin{array}{ccc}
\varepsilon_{z z}^{P_{i}} & 0 & 0 \\
0 & \varepsilon_{z z}^{P_{i}} & 0 \\
0 & 0 & \varepsilon_{x x}^{P_{i}}+\varepsilon_{y y}^{P_{i}}+2 \varepsilon_{z z}^{P_{i}}
\end{array}\right)\left(\begin{array}{c}
\sin \theta_{d} \cos \phi_{d} \\
\sin \theta_{d} \sin \phi_{d} \\
\cos \theta_{d}
\end{array}\right) .
\end{aligned}
$$

Then, we plug the strain induced by the incident $\mathrm{P}$-wave in equation A-2 into equation C-2 and, finally, we obtain the P-P-radiation pattern of the PDW for the $V_{\mathrm{P} 3}$ perturbation as a function of incidence and diffraction angles as follows:

$$
\begin{aligned}
& \mathbb{R}_{V_{\mathrm{P} 3}, \mathrm{CP}-\mathrm{I}}^{\mathrm{P}-\mathrm{P}}\left(\theta_{i}, \phi_{i}, \theta_{d}, \phi_{d}\right) \\
& \quad=\frac{\rho V_{\mathrm{P}}}{h}\left[\sin ^{2} \theta_{d} \cos ^{2} \theta_{i}+\cos ^{2} \theta_{d}\left(\sin ^{2} \theta_{i}+2 \cos ^{2} \theta_{i}\right)\right] .
\end{aligned}
$$

In the same manner, the P-SV-radiation pattern of the PDW for the $V_{\mathrm{P} 3}$ perturbation can be derived as follows:

$$
\begin{aligned}
& \mathbb{R}_{V_{\mathrm{P} 3}, \mathrm{CP}-\mathrm{I}}^{\mathrm{P}-\mathrm{SV}}\left(\theta_{i}, \phi_{i}, \theta_{d}, \phi_{d}\right) \\
& \quad=\mathbf{e}_{\mathrm{SV}_{d}}^{T} \mathbf{M}_{V_{\mathrm{P} 3}, \mathrm{CP}-\mathrm{I}}^{P_{i}} \mathbf{e}_{P_{d}}=\frac{-\rho V_{\mathrm{P}}}{h}\left(\sin \theta_{d} \cos \theta_{d}\right) .
\end{aligned}
$$

As an example, in Figure C-1, we display the diffraction patterns of the PDW for the $V_{\mathrm{P} 3}$ perturbation we obtained numerically. We take snapshots of the PDW for each parameter from a single explosive source, which generates only incident $\mathrm{P}$-wave, located in the middle of the surface with $0.8 \mathrm{~km}$ depth (red circles). We chose 13 grid points (eight on the source and receiver plane, four at $45^{\circ}$ incidence angle in inline and crossline and one at normal incidence), which have the same distance from the source but their incidence angles vary so that we can observe how the source mechanism of the model parameter changes depending on the incidence angles. We also display the reflection raypath as dashed lines, which corresponds to the transmission raypath on the source-receiver plane, so that we can observe how much energy of reflected P- and SV-waves of the PDW for the $V_{\mathrm{P} 3}$ perturbation we record on the surface. We record all three components and we plot the square root of the displacement vector of the PDW that exactly corresponds to the total radiation response $\left(\mathbb{R}_{\text {Total }}\right.$; in equation 18$)$.

In equations C-3 and C-4, we observe that P-P- and P-SV-waves are independent of the azimuth angles. For this reason, on the $x y$-plane in Figure C-1, we observe that the P-P- and P-SV-radiation patterns are also azimuth independent. However, the P-P-waves are dependent on the incidence angle $\theta_{i}$. We observe that, in equation C-2, the virtual source of $V_{\mathrm{P} 3}$ loses its horizontal force at a large incidence angle because vertical normal strain goes to zero. For this reason, in the $x z$ - and $y z$-planes of Figure C-1, the P-P- 


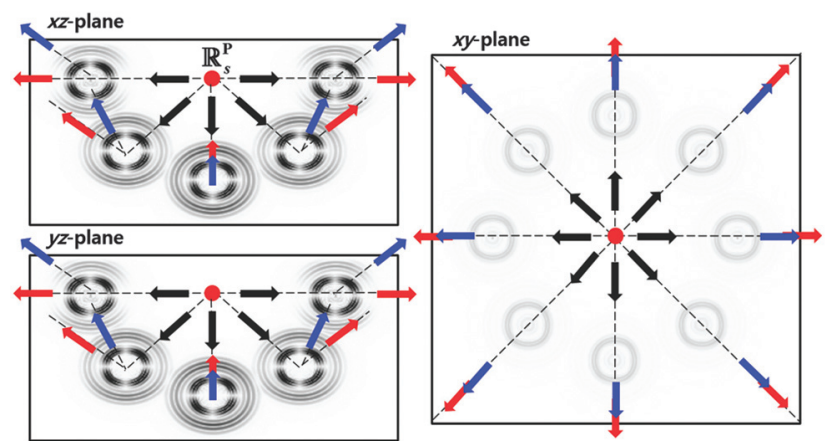

Figure C-1. An example ( $V_{\mathrm{P} 3}$ in CP-I; Figure $\left.13 \mathrm{c}\right)$ of how to interpret the diffraction patterns in Figures 2, 8, and 13. The red circle indicates an explosive source and dashed lines indicate the reflection (or transmission at $180^{\circ}$ opening angle) raypaths. The black, red, and blue arrows indicate the incident $\mathrm{P}$-wave, reflected (or transmitted) P-wave, and reflected (or transmitted) S-wave, respectively, which are propagated following Snell's law. These diffraction patterns show how much energy propagates along the reflection raypath and how much energy propagate out of the reflection raypath depending on the parameters. The $x z^{-}(y=3 \mathrm{~km}), y z_{-}$ $(x=3 \mathrm{~km})$, and $x y-(z=0.8 \mathrm{~km})$ planes cross the source position.

waves show a nearly isotropic diffraction pattern at the vertical incidence, but they show a vertically dominated diffraction pattern at a horizontal incidence. On the other hand, the P-SV-waves are also independent of the incidence angles as equation C-4 shows. For this reason, we observe incidence angle-independent radiation patterns for the P-SV-wave in the PDW for the $V_{\mathrm{P} 3}$ perturbation in CP-I. In the same manner, we can interpret the diffraction patterns of each parameter in each parameterization that are displayed in Figures 2, 8 , and 13 .

\section{REFERENCES}

Aki, K., and P. G. Richards, 1980, Quantitative seismology: Freeman Alkhalifah, T., 2003, An acoustic wave equation for orthorhombic anisotropy: Geophysics, 68, 1169-1172, doi: 10.1190/1.1598109.

Alkhalifah, T., 2015a, Full waveform inversion in an anisotropic world: Where are the parameters hiding?: EAGE publication, Education Tour Series.

Alkhalifah, T., 2015b, Scattering-angle based filtering of the waveform inversion gradients: Geophysical Journal International, 200, 363-373, doi: 10.1093/gji/ggu379.

Alkhalifah, T., 2016, Insights into the data dependency on anisotropy: An inversion prospective: Geophysical Prospecting, 64, 505-513, doi: 10 $.1111 / 1365-2478.12345$.

Alkhalifah, T., and R.-E. Plessix, 2014, A recipe for practical fullwaveform inversion in anisotropic media: An analytical parameter resolution study: Geophysics, 79, no. 3, R91-R101, doi: 10.1190/ geo2013-0366.1.

Bergslid, T. S., E. B. Raknes, and B. Arntsen, 2015, The influence of anisotropy on elastic full-waveform inversion: 85th Annual International Meeting, SEG, Expanded Abstracts, 1425-1429.

Gholami, Y., R. Brossier, S. Operto, A. Robodetti, and J. Virieux, 2013a, Which parameterization is suitable for acoustic vertical transverse isotropic full waveform inversion? Part 1: Sensitivity and tradeoff analysis: Geophysics, 78, no. 2, R81-R105, doi: 10.1190/ geo2012-0204.1.

Gholami, Y., R. Brossier, S. Operto, A. Robodetti, and J. Virieux, 2013b, Which parameterization is suitable for acoustic vertical transverse isotropic full waveform inversion? Part 2: Synthetic and real data case studies from Valhall: Geophysics, 78, no. 2, R107-R124, doi: 10.1190/ geo2012-0203.1
Graves, R. W., 1996, Simulating seismic wave propagation in 3D elastic media using staggered-grid finite differences: Bulletin of the Seismological Society of America, 86, 1091-1106.

Jost, M. L., and R. B. Herrmann, 1989, A student's guide to and review of moment tensors: Seismological Research Letters, 60, 37-57.

Kamath, N., and I. Tsvankin, 2013, Gradient computation for elastic fullwaveform inversion in 2D VTI media: 83rd Annual International Meeting, SEG, Expanded Abstracts, 1073-1078.

Kamath, N., and I. Tsvankin, 2016, Elastic full-waveform inversion for VTI media: Methodology and sensitivity analysis: Geophysics, 81, no. 2, C53-C68, doi: 10.1190/geo2014-0586.1.

Köhn, D., D. D. Nil, A. Kurzmann, A. Przebindowska, and T. Bohlen, 2012, On the influence of model parametrization in elastic full waveform tomography: Geophysical Journal International, 191, 325-345, doi: 10 $.1111 / \mathrm{j} .1365-246 \mathrm{X} .2012 .05633 . x$.

Köhn, D., O. Hellwig, D. D. Nil, and W. Rabbel, 2015, Waveform inversion in triclinic anisotropic media - A resolution study: Geophysical Journal International, 201, 1642-1656, doi: 10.1093/gji/ggv097.

Koo, J. M., H.-Y. Lee, W. Jeong, D.-J. Min, and H. S. Yoo, 2010, Frequencydomain elastic full waveform inversion for VTI media: 72nd Annua International Conference and Exhibition, EAGE, Extended Abstracts, P381.

Lee, H.-Y., J. M. Koo, D.-J. Min, B.-D. Kwon, and H. S. Yoo, 2010, Frequency-domain elastic full waveform inversion for VTI media: Geophysical Journal International, 183, 884-904, doi: 10.1111/j.1365-246X.2010 .04767.x.

Masmoudi, N., and T. Alkhalifah, 2016, A new parameterization for waveform inversion in acoustic orthorhombic media: Geophysics, 81, no. 4 R157-R171, doi: 10.1190/geo2015-0635.1.

Oh, J. W., and D. J. Min, 2014, Multi-parametric FWI using a new parameterization for elastic VTI media: 76th Annual International Conference and Exhibition, EAGE, Extended Abstracts, doi: 10.3997/2214-4609 20141084.

Oh, J. W., and T. Alkhalifah, 2015, Study on orthorhombic parameters for 3D elastic full waveform inversion: 85 th Annual International Meeting, SEG, Expanded Abstracts, 1404-1408.

Oh, J. W., and T. Alkhalifah, 2016, The scattering potential of partial derivative wavefields in 3D elastic orthorhombic media: An inversion prospective: Geophysical Journal International, 206, 1740-1760, doi: 10.1093/ gji/ggw238.

Operto, S., Y. Gholami, V. Prieux, A. Ribodetti, R. Brossier, L. Métivier, and J. Virieux, 2013, A guided tour of multiparameter full-waveform inversion with multicomponent data: From theory to practice: The Leading Edge, 32, 1040-1054, doi: 10.1190/tle32091040.1.

Plessix, R.-É., and Q. Cao, 2011, A parametrization study for surface seismic full waveform inversion in an acoustic vertical transversely isotropic medium: Geophysical Journal International, 185, 539-556, doi: 10.1111/j .1365-246X.2011.04957.x.

Prieux, V., R. Brossier, S. Operto, and J. Virieux, 2013a, Multiparameter full waveform inversion of multicomponent ocean-bottom-cable data from the Valhall field. Part 1: Imaging compressional wave speed, density and attenuation: Geophysical Journal International, 194, 1640-1664, doi: 10 .1093/gii/ggt177.

Prieux, V., R. Brossier, S. Operto, and J. Virieux, 2013b, Multiparameter full waveform inversion of multicomponent ocean-bottom-cable data from the Valhall field. Part 2: Imaging compressive-wave and shear-wave velocities: Geophysical Journal International, 194, 1665-1681, doi: 10 .1093/gji/ggt178.

Ramos-Martinez, J., S. Crawley, Z. Zou, A. A. Valenciano, L. Qiu, and N. Chemingui, 2016, A robust gradient for long wavelength FWI updates: 78th Annual International Conference and Exhibition, EAGE, Extended Abstracts, doi: 10.3997/2214-4609.201601536.

Song, X., and T. Alkhalifah, 2013, Modeling of pseudo-acoustic P-waves in orthorhombic media with a lowrank approximation: Geophysics, 78, no. 4, C33-C40, doi: 10.1190/geo2012-0144.1.

Tarantola, A., 1986, A strategy for nonlinear elastic inversion of seismic reflection data: Geophysics, 51, 1893-1903, doi: 10.1190/1.1442046.

Thomsen, L., 1986, Weak elastic anisotropy: Geophysics, 51, 1954-1966, doi: 10.1190/1.1442051.

Tsvankin, I., 1997, Anisotropic parameters and P-wave velocity for orthorhombic media: Geophysics, 62, 1292-1309, doi: 10.1190/1.1444231.

Virieux, J., and S. Operto, 2009, An overview of full-waveform inversion in exploration geophysics: Geophysics, 74, no. 6, WCC1-WCC26, doi: 10 $.1190 / 1.3238367$.

Wu, R., and K. Aki, 1985, Scattering characteristics of elastic waves by an elastic heterogeneity: Geophysics, 50, 582-595, doi: 10.1190/1.1441934

Wu, R., and K. Aki, 1988, Introduction: Seismic wave scattering in threedimensionally heterogeneous earth: Pure and Applied Geophysics, 128, 1-6, doi: 10.1007/BF01772587. 